\title{
The Orion Protostellar Explosion and Runaway Stars Revisited: Stellar Masses, Disk Retention, and an Outflow from the Becklin-Neugebauer Object
}

\author{
John Bally ${ }^{1}$ (i), Adam Ginsburg ${ }^{2}$ (i) Jan Forbrich $^{3}$ (1D), and Jaime Vargas-González ${ }^{3}$ \\ ${ }^{1}$ Center for Astrophysics and Space Astronomy, Astrophysical and Planetary Sciences Department, University of Colorado, UCB 389 Boulder, CO 80309 , USA \\ john.bally@ colorado.edu \\ ${ }^{2}$ Department of Astronomy, University of Florida, Bryant Space Science Center, Stadium Road, Gainesville, FL 32611, USA \\ ${ }^{3}$ Centre for Astrophysics Research, School of Physics, Astronomy and Mathematics, Hertfordshire University, College Lane, Hatfield, AL10 9AB, UK \\ Received 2019 July 19; revised 2019 December 17; accepted 2019 December 23; published 2020 February 5
}

\begin{abstract}
The proper motions of the three stars ejected from Orion's OMC1 cloud core are combined with the requirement that their center of mass is gravitationally bound to OMC1 to show that radio source I (Src I) is likely to have a mass around $15 M_{\odot}$ consistent with recent measurements. Src I, the star with the smallest proper motion, is suspected to be either an astronomical-unit-scale binary or a protostellar merger remnant produced by a dynamic interaction $\sim 550 \mathrm{yr}$ ago. Near-infrared $2.2 \mu \mathrm{m}$ images spanning $\sim 21 \mathrm{yr}$ confirm the $\sim 55 \mathrm{~km} \mathrm{~s}^{-1}$ motion of "source $\mathrm{x}$ " (Src $\mathrm{x}$ ) away from the site of stellar ejection and point of origin of the explosive OMC1 protostellar outflow. The radial velocities and masses of the Becklin-Neugebauer (BN) object and Src I constrain the radial velocity of Src $\mathrm{x}$ to be $V_{\mathrm{LSR}}=-28 \pm 10 \mathrm{~km} \mathrm{~s}^{-1}$. Several high proper-motion radio sources near BN, including Zapata 11 ([ZRK2004] 11) and a diffuse source near IRc 23, may trace a slow bipolar outflow from BN. The massive disk around Src I is likely the surviving portion of a disk that existed prior to the stellar ejection. Though highly perturbed, shocked, and reoriented by the $N$-body interaction, enough time has elapsed to allow the disk to relax with its spin axis roughly orthogonal to the proper motion.
\end{abstract}

Unified Astronomy Thesaurus concepts: Plasma jets (1263); Star formation (1569); Massive stars (732); Runaway stars (1417); Stellar jets (1607); Stellar-interstellar interactions (1576); Stellar feedback (1602); Circumstellar disks (235); Protostars (1302)

\section{Introduction}

The Orion OMC1 cloud core located a few tenths of a parsec behind the Orion Nebula contains the nearest site of ongoing massive star formation (O'Dell 2001; Bally 2016). The outflow emerging from $\mathrm{OMC} 1$ has a kinetic energy of $(2-4) \times 10^{47} \mathrm{erg}$ today and exhibits a remarkable explosive morphology (Zapata et al. 2009; Bally et al. 2011, 2015, 2017; Bally 2016). This outflow originated from within a few arcseconds of the location from which three stars were ejected with speeds of $\sim 10$ to $55 \mathrm{~km} \mathrm{~s}^{-1}$ about $550 \mathrm{yr}$ ago. The Becklin-Neugebauer (BN) object has a proper motion of $\sim 26 \mathrm{~km} \mathrm{~s}^{-1}$ toward the northwest, radio source Src I moves at $\sim 10 \mathrm{~km} \mathrm{~s}^{-1}$ toward the south, and near-infrared source $\operatorname{Src} x$ moves at $\sim 55 \mathrm{~km} \mathrm{~s}^{-1}$ toward the southeast (Dzib et al. 2017; Luhman et al. 2017; Rodríguez et al. 2017). The stellar ejection and the explosion in the gas appear to have been triggered by the same event, likely caused by an $\mathrm{N}$-body stellar interaction that led to either the formation of an astronomical-unit-scale binary or a stellar merger that released at least $10^{48} \mathrm{erg}$ of gravitational potential energy required to power the explosion and the acceleration of runaway stars (Zapata et al. 2009; Goddi et al. 2011; Bally et al. 2017; Farias \& Tan 2018).

Before 2017, infrared source $\mathrm{n}$ ( $\mathrm{Src} \mathrm{n}$ ) was thought to be a runaway star from $\mathrm{OMC} 1$ because radio emission from this object exhibited high proper motions. However, Rodríguez et al. (2017) showed that radio proper motions were biased by the ejection of a one-sided radio jet around 2006, implying that Src n may not have a measurable proper motion in the Orion reference frame. However, near-infrared Hubble Space Telescope (HST) images revealed that Lonsdale et al.'s (1982) "source x" (Src x) has a $\sim 55 \mathrm{~km} \mathrm{~s}^{-1}$ proper motion away from the explosion center (Luhman et al. 2017).
$\mathrm{SiO}$ masers and other emission lines tracing the Src I disk show that it has a radial velocity $V_{\mathrm{LSR}}=5.0 \mathrm{~km} \mathrm{~s}^{-1}$ (Plambeck \& Wright 2016). Ginsburg et al. $(2018,2019)$ found $V_{\mathrm{LSR}}=$ $5.5 \mathrm{~km} \mathrm{~s}^{-1}$ using thermal emission lines. The OMC1 core has a radial velocity $V_{\mathrm{OMC} 1}=9.0-9.5 \mathrm{~km} \mathrm{~s}^{-1}$. Thus, we adopt a value of $V_{r}(I)=-4 \pm 0.5 \mathrm{~km} \mathrm{~s}^{-1}$ for the radial velocity of Src I in the Orion reference frame. Hydrogen recombination lines from BN show that it has a radial velocity $V_{\mathrm{LSR}}=23.2 \pm 0.5 \mathrm{~km} \mathrm{~s}^{-1}$, implying a $V_{r}(\mathrm{BN})=+14.2 \pm 0.5 \mathrm{~km} \mathrm{~s}^{-1}$ in the Orion reference frame (Plambeck et al. 2013). These radial velocity measurements are combined with proper motion measurements of the three ejected stars to estimate their velocity vectors.

The mass of the BN object, thought to be an early B star, has been estimated to be in the range of $10-15 M_{\odot}$ based on spectroscopy, its luminosity, and the presence of hydrogen recombination lines and free-free emission from a hypercompact H II region (Scoville et al. 1983; Jiang et al. 2005; Plambeck et al. 2013). The mass of Src $\mathrm{x}$ is estimated to be roughly $3 M_{\odot}$ based on its IR spectrum (Luhman et al. 2017).

Unfortunately, Src I is so embedded that it is only visible at submillimeter to radio wavelengths. Thus, its mass cannot be determined from its stellar spectrum. However, it is surrounded by a circumstellar disk whose rotation curve has been used to estimate its mass dynamically. Measurements with an angular resolution as high as 0.22 resulted in masses around 5-8 $M_{\odot}$ using the so-called centroid-of-channels method (Kim et al. 2008, 2019; Matthews et al. 2010; Hirota et al. 2014, 2017; Plambeck \& Wright 2016).

Ginsburg et al. (2018) used ALMA to study the disk rotation curve with the highest angular resolution achieved thus far, $\sim 0$ " 03 . Three emission lines were used: the $232.6867 \mathrm{GHz}$ $\mathrm{H}_{2} \mathrm{O} 5_{5,0}-6_{4,3}$ transition whose upper state is $3461.9 \mathrm{~K}$ above 
ground and transitions at 217.980 and $232.511 \mathrm{GHz}$ from (at the time of publication) an unknown carrier. Fitting the maximum radial velocity at each position in the nearly edgeon disk, Ginsburg et al. (2018) derived a mass of $\sim 15 \pm 2 M_{\odot}$ for Src I. The mass measurement was verified using radiative transfer modeling analogous to that used by Plambeck \& Wright (2016). Ginsburg et al. (2019) show that the two unknown lines are transitions of salt, $\mathrm{NaCl}, v=2, J=17-16$ and $v=1, J=18-17$, respectively, with upper states 1128 and $626 \mathrm{~K}$ above ground.

Farias \& Tan (2018) presented $\sim 10^{7} N$-body scattering simulations of the stellar dynamical ejection. They found that a binary-binary interaction has the highest probability of leading to the observed configuration of three runaway stars, Src I, BN, and Src x. In their model, they consider Src I to be a compact binary. They also explore the system CoM velocity as a function of the initial stellar masses and motions. They show that the mass of Src I (total mass of a binary or a single star) is likely to be between 5 and $25 M_{\odot}$ with a most likely value near $14 M_{\odot}$.

In this paper, we present archival observations that provide additional constraints on the motions of stars in the OMC1 region. We use the measured proper motions, a range of estimated masses for $\mathrm{BN}$ and $\mathrm{Src} \mathrm{x}$, and the requirement that the center of mass $(\mathrm{CoM})$ of the stars move with a speed less than the gravitational escape speed from OMC1 to show that Src I must have a mass around $15 M_{\odot}$. We use the measured radial velocities of $\mathrm{BN}$ and $\mathrm{Src} I$ to predict the radial velocity of Src $\mathrm{x}$, which thus far has not been measured.

The distance to OMC1 had been determined to be $D \approx$ $414 \pm 7$ pc using radio parallaxes to young stars exhibiting nonthermal emission (Menten et al. 2007) and the $\mathrm{H}_{2} \mathrm{O}$ masers in the outflow yielding $D \approx 418 \pm 6$ pc (Kim et al. 2008). More recent parallax measurements to radio-continuum-emitting stars in the Orion A molecular cloud, which hosts OMC1, yield a distance of $388 \pm 5$ pc (Kounkel et al. 2017). Several shocks powered by the OMC1 outflow protrude into the Orion Nebula, indicating that the OMC1 core must be only a few tenths of a parsec behind the nebula's ionization front and not far behind most of the stars measured by Kounkel et al. (2017). We adopt a distance of 400 pc to the OMC1 core, the explosive outflow, and associated stars and use this value in all calculations.

In addition to $\mathrm{Src} \mathrm{I}$ and $\mathrm{BN}$, several additional compact radio continuum features near $\mathrm{BN}$ exhibit proper motions away from the explosion and stellar ejection center (Dzib et al. 2017). A 1" diameter radio source located $7 !$ ! 7 east of $\mathrm{BN}$ at position angle $\mathrm{PA} \sim 77^{\circ}$ and dubbed "IRc 23" was found to move with a velocity of $\sim 39 \mathrm{~km} \mathrm{~s}^{-1}$ toward PA $\sim 9^{\circ}$ (Dzib et al. 2017). IRc 23 is actually an infrared source (Shuping et al. 2004) located about 1". 3 northeast of this radio feature and is associated with a bipolar disk shadow in images at $\lambda=1.64$ and $2.12 \mu \mathrm{m}$ (Bally et al. 2015). The source [ZRK] 11 (Zapata et al. 2004), referred to as Zapata 11 in the rest of this manuscript, is located $\sim 2^{\prime \prime}$ west of BN. According to Dzib et al. (2017), Zapata 11 has a velocity of $28 \mathrm{~km} \mathrm{~s}^{-1}$ toward $\mathrm{PA} \sim 308^{\circ}$, similar to the motion of $\mathrm{BN}$ itself.

Neither of these slightly extended radio sources have X-ray, infrared, or millimeter-wave counterparts. Thus, they are unlikely to contain stars. These radio features are likely to be ionized ejecta produced by the $\mathrm{OMC} 1$ explosion or powered by an outflow from
BN itself. Therefore, they probably have negligible mass and will be ignored in our center-of-momentum analysis.

Figure 1 shows the central part of the OMC1 explosion in an image obtained through a $\sim 1 \%$ bandpass narrowband filter transmitting the $2.12 \mu \mathrm{m} \mathrm{S}(1)$ rovibrational transition of $\mathrm{H}_{2}$. This image, taken from Bally et al. (2015), has a resolution of $\sim 0$." 06 and is reproduced here to show the proper motion of Src $\mathrm{x}$, which was not known to be a runaway star in 2015 . The locations and proper motions of the three ejected stars are indicated by the cyan labels and yellow vectors, respectively.

\section{Observations and Analysis}

Multiepoch infrared images obtained at wavelengths ranging from 2 to $12 \mu \mathrm{m}$ and listed in Table 1 are used to confirm the $\sim 55 \mathrm{~km} \mathrm{~s}^{-1}$ proper motion of Src $\mathrm{x}$ and to place limits on the motion of Src n. The FITS images were registered to the 0".06 resolution laser-guide-star adaptive optics 2013 epoch image published in Bally et al. (2015) and shown in Figure 1 using two dozen stars contained within the subregion of the image shown in Figure 2. IRAF routines GEOMAP and GEOTRAN were used to match the infrared images listed in Table 1 to the 2013 data.

The $\sim 21 \mathrm{yr}$ interval between the Allen \& Burton (1993) images obtained in 1992 and the Bally et al. (2017) images obtained in 2013 confirm the high proper motion of Src x. The Allen \& Burton (1993) image was a mosaic built using a firstgeneration 58 by 62 pixel InSb array, and the image pointspread function (PSF) is about $2^{\prime \prime}$ in diameter. The proper motion uncertainty is about $20 \mathrm{~km} \mathrm{~s}^{-1}$ in amplitude and $20^{\circ}$ in position angle.

The highest quality ground-based images for proper motion determinations are the 1999 epoch narrowband $\mathrm{H}_{2}$ Subaru image from Kaifu et al. (2000), which has a 0."5 PSF, and the 2013 epoch narrowband $\mathrm{H}_{2}$ Gemini South image from Bally et al. (2015), which has a 0."06 PSF. Both data sets were built from observations taken over a range of dates: $\mathrm{MJD}=51189$ to 51191 for the 1999 epoch Subaru image and MJD $=56291$ to 56351 for the 2013 epoch Gemini South image. The time interval between these images is $5131 \pm 31$ days $\left(\approx 4.43 \times 10^{8} \mathrm{~s}\right)$. Figure 2 shows a difference image obtained by subtracting the registered 1999 image from the 2013 image.

Only a few stars are in common between the Smith et al. (2005) $11.7 \mu \mathrm{m}$ image, which has a 0!3 PSF, and the Bally et al. (2015) image with a $9 \mathrm{yr}$ interval. However, comparison of the registered images confirms the motion of Src $\mathrm{x}$ with a large uncertainty in amplitude and position angle of the proper motion vector.

Dzib et al. (2017) list two additional high-velocity sources in their $\sim 5 \mathrm{GHz}$ radio proper motion analysis, in their table designated Zapata 11 and "IRc 23." We compared the $6 \mathrm{GHz}$ radio radio continuum data from epoch 2012 JVLA observations, previously published by Forbrich et al. (2016), to the above infrared images to show that these fast moving features near BN do not contain compact sources between 1.6 and $11.7 \mu \mathrm{m}$. Additionally, we use the ALMA data obtained under program 2016.1.00165.S to show that Zapata 11 and IRc 23 do not contain compact sources at millimeter wavelengths. Thus, as discussed below, these objects are likely to trace ejecta from $\mathrm{BN}$ rather than stars and will therefore not be included in the motion of the CoM calculations.

We use $6 \mathrm{GHz}$ JVLA images obtained on 2016 November 27 (J. Forbrich et al. 2020, in preparation) together with the $6 \mathrm{GHz}$ 


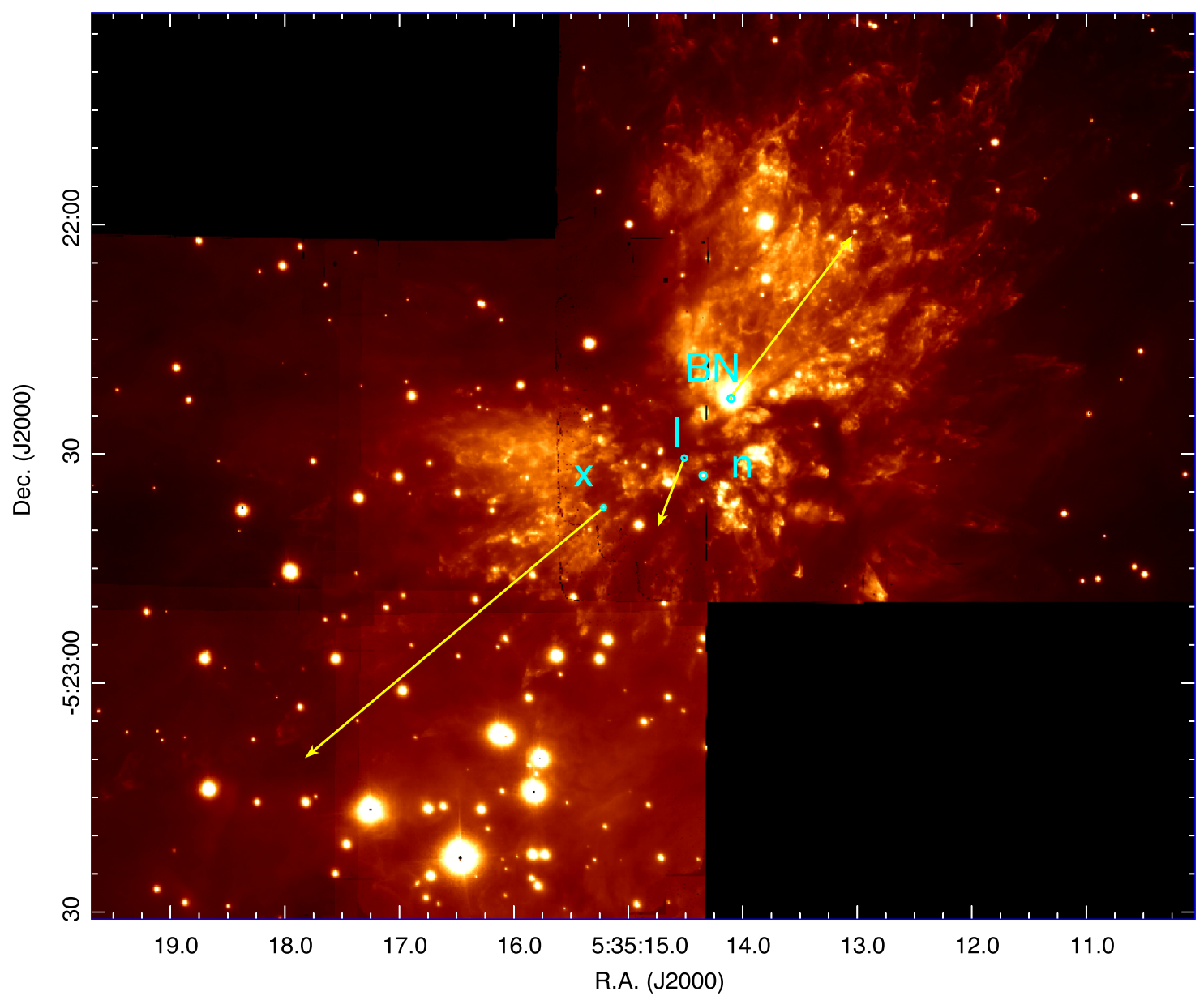

Figure 1. A $2.12 \mu \mathrm{m}$ narrowband $\mathrm{H}_{2}$ image from Bally et al. (2015) showing $\mathrm{Src}$ x, Src I, BN, and Src $\mathrm{n}$. The yellow arrows show the proper motions of the three ejected stars with lengths corresponding to the motions over the next $2000 \mathrm{yr}$. The vectors shown are based on the motions measured by Rodríguez et al. (2017) for the BN object and Src I, and by Luhman et al. (2017) for Src x. The Trapezium cluster of massive stars in the center of the Orion Nebula are located near the bottom of the image.

Table 1

Summary of Near-IR Data Used in This Study

\begin{tabular}{|c|c|c|c|c|}
\hline Telescope & Date & MJD & Wavelength & References \\
\hline AAT & 1992 Aug 15 & 48849 & $1.644,2.12 \mu \mathrm{m}$ & Allen \& Burton (1993) \\
\hline Subaru & 1999 Jan $11-13$ & $\sim 51190$ & $2.12 \mu \mathrm{m}$ & Kaifu et al. (2000) \\
\hline APO $3.5 \mathrm{~m}$ & 2004 Nov 22 & 53331 & $2.12 \mu \mathrm{m}$ & Bally et al. (2011) \\
\hline Gemini-S & 2012 Dec 30-2013 Feb 28 & $\sim 56321$ & $1.644,2.12 \mu \mathrm{m}$ & Bally et al. (2015) \\
\hline
\end{tabular}

VLA data observed on 2012 September 30 to October 5 (Forbrich et al. 2016) to measure new proper motions for the radio sources near BN. In 2012, $30 \mathrm{hr}$ of on-source data were acquired over five days in two $1 \mathrm{GHz}$ wide bands centered at 4.7 and $7.3 \mathrm{GHz}$. The combined images have an rms noise level of $3 \mu \mathrm{Jy}$ beam $^{-1}$ and a synthesized beam diameter 0 !' $19 \times 0$ " 30 at P.A. $30^{\circ}$. In 2016, only $4 \mathrm{hr}$ of on-source data were acquired in a configuration identical to the one used in 2012. The interval between these observations is $1.31 \times 10^{8} \mathrm{~s}(4.16 \mathrm{yr})$.

\section{Results}

Table 2 lists the motions of the three runaway stars as well as two radio sources near $\mathrm{BN}$ which were listed in Dzib et al. (2017) in the Orion reference. We use a distance of $400 \mathrm{pc}$ for the conversion of proper motions (given in milliarcseconds per year) to velocity vectors. For Src I and BN, the most reliable proper motion determinations are those of Rodríguez et al. (2017); for Src x, the best determinations are the HST-based measurements of Luhman et al. (2017). Table 3 lists what we deem as the best values for the motions of the ejected stars.

\subsection{Constraints on Stellar Masses}

We used these measurements and their errors to compute the motion of the CoM on the plane of the sky of the three ejected stars as functions of their masses, $M_{\mathrm{BN}}, M_{I}$, and $M_{x}$. If the proper motion vectors of the three stars in the Orion reference frame are given by $V_{\mathrm{BN}}, V_{I}$, and $V_{x}$, respectively, the CoM of 


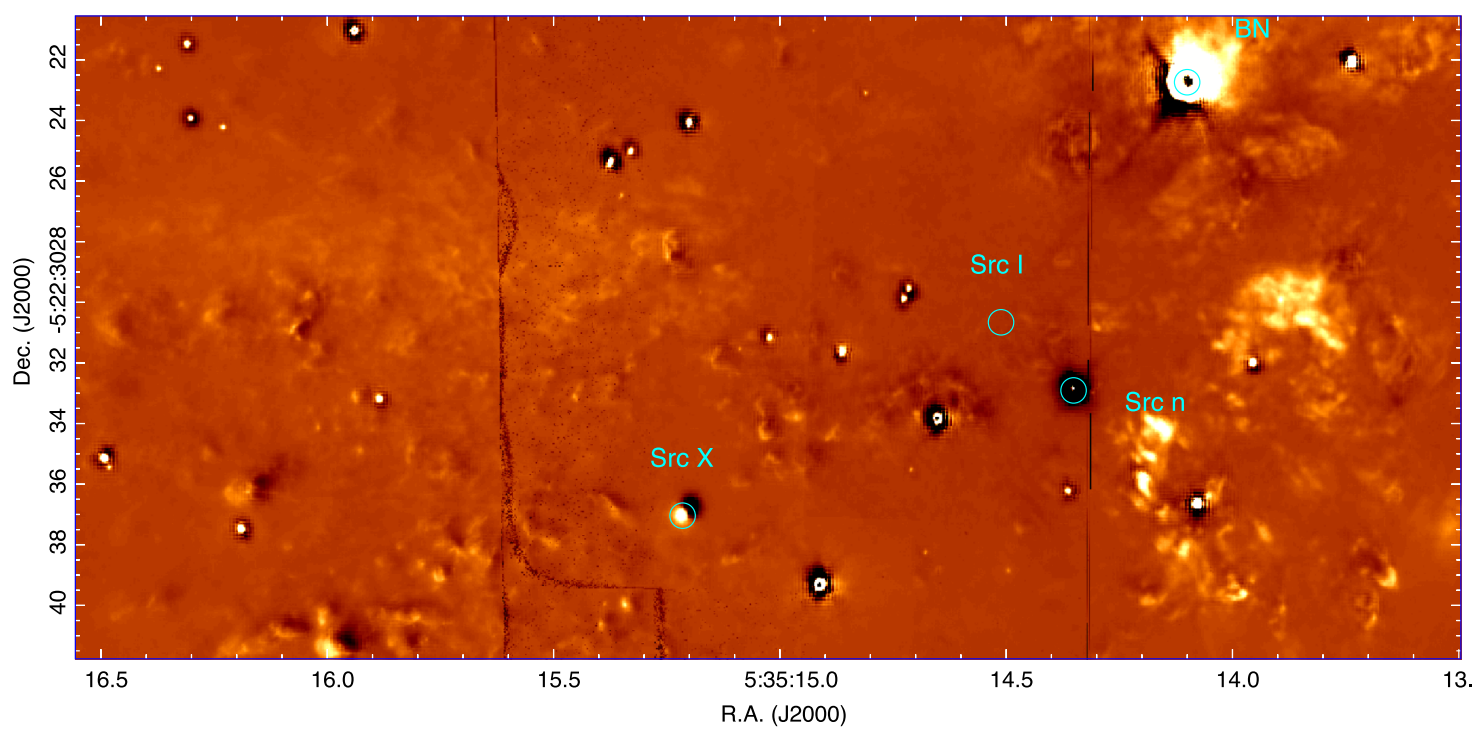

Figure 2. A difference image formed from the 2013 epoch $2.12 \mu \mathrm{m}$ narrowband $\mathrm{H}_{2}$ image from Bally et al. (2015) and the 1999 epoch Subaru image from Kaifu et al. (2000).

Table 2

Positions and Proper Motions of the Runaway Stars in $\mathrm{OMC} 1$

\begin{tabular}{|c|c|c|c|c|c|c|c|}
\hline Source & $\begin{array}{l}\text { R.A. } \\
\text { (J2000) }\end{array}$ & $\begin{array}{l}\text { Decl. } \\
(\mathrm{J} 2000)\end{array}$ & $\begin{array}{c}\mu(\alpha) \cos (\delta) \\
(\text { mas })\end{array}$ & $\begin{array}{l}\mu(\delta) \\
\text { (mas) }\end{array}$ & $\begin{array}{c}V \\
\left(\mathrm{~km} \mathrm{~s}^{-1}\right)\end{array}$ & $\begin{array}{l}\text { P.A. } \\
\left({ }^{\circ}\right)\end{array}$ & Comments \\
\hline \multirow{6}{*}{$\overline{\mathrm{BN}}$} & $5: 35: 14.110$ & $-5: 22: 22.70$ & $-8.1 \pm 0.4$ & $10.8 \pm 0.4$ & $25.6 \pm 0.8$ & $323 \pm 2$ & Rodríguez et al. (2017) \\
\hline & & & $-6.9 \pm 1.4$ & $10.3 \pm 1.4$ & $23.5 \pm 4$ & $326 \pm 10$ & Luhman et al. (2017) \\
\hline & & & $-4.3 \pm 1.1$ & $12.7 \pm 1.1$ & $25.4 \pm 2.1$ & $341 \pm 5$ & Goddi et al. (2011) \\
\hline & & & $-4.6 \pm 1.1$ & $11.2 \pm 1.1$ & $23.0 \pm 2.1$ & $338 \pm 5$ & Transformed to Dzib ONC frame \\
\hline & & & $-7.2 \pm 2.7$ & $12.2 \pm 1.9$ & $26.9 \pm 3.3$ & $330 \pm 8$ & Kim et al. (2019) \\
\hline & & & $-7.95 \pm 2$ & $9.45 \pm 2$ & $23.4 \pm 5$ & $320 \pm 15$ & this work \\
\hline \multirow[t]{3}{*}{ Src I } & $5: 35: 14.520$ & $-5: 22: 30.65$ & $1.8 \pm 0.4$ & $-4.6 \pm 0.4$ & $9.4 \pm 0.8$ & $159 \pm 5$ & Rodríguez et al. (2017) \\
\hline & & & $5.5 \pm 1.1$ & $-1.9 \pm 1.1$ & $11.0 \pm 2.3$ & $109 \pm 12$ & Goddi et al. (2011) \\
\hline & & & $5.2 \pm 1.1$ & $-3.4 \pm 1.1$ & $11.8 \pm 2.3$ & $123 \pm 12$ & Transformed to Dzib ONC frame \\
\hline \multirow[t]{3}{*}{$\operatorname{Src} x$} & $5: 35: 15.215$ & $-5: 22: 37.04$ & $23.3 \pm 1.4$ & $-17.4 \pm 1.4$ & $55.1 \pm 4$ & $127 \pm 3$ & Luhman et al. (2017) \\
\hline & & & $26.8 \pm 1.5$ & $-18.4 \pm 1.5$ & $61.6 \pm 5$ & $125 \pm 4$ & Kim et al. (2019) \\
\hline & & & $20.2 \pm 3$ & $-12.0 \pm 3$ & $44.6 \pm 8$ & $120 \pm 6$ & this work \\
\hline \multirow[t]{5}{*}{$\operatorname{Src} n$} & $5: 35: 14.361$ & $-5: 22: 32.75$ & $0.0 \pm 3$ & $0.0 \pm 3$ & $0.0 \pm 8$ & $\ldots$ & this work \\
\hline & & & $1.9 \pm 1.0$ & $1.0 \pm 0.7$ & $4.0 \pm 3$ & $62 \pm 40$ & Kim et al. (2019) \\
\hline & & & $-1.8 \pm 1.4$ & $-2.5 \pm 1.4$ & $5.8 \pm 3$ & $215 \pm 40$ & Luhman et al. (2017) \\
\hline & & & $<3$ & $<3$ & $<8$ & $\ldots$ & Goddi et al. (2011) \\
\hline & & & $0.0 \pm 0.9$ & $-7.8 \pm 0.4$ & $14.8 \pm 1.2$ & $180.0 \pm 4$ & jet from n Rodríguez et al. (2017) \\
\hline SW1 (Zapata 11) & $05: 35: 14.019$ & $-5: 22: 23.25$ & $-11.8 \pm 5.6$ & $9.3 \pm 2.3$ & $28.4 \pm 11$ & $308 \pm 20$ & PM from Dzib et al. (2017) \\
\hline SW1 & 05:35:14.107 & $-5: 22: 23.20$ & $-22.1 \pm 9.0$ & $5.3 \pm 5.0$ & $43.1 \pm 20$ & $283 \pm 30$ & PM from this work \\
\hline SW2 (152) & $05: 35: 13.953$ & $-5: 22: 23.82$ & $17.2 \pm 9.4$ & $-8.8 \pm 5.8$ & $36.6 \pm 21$ & $-117 \pm 30$ & PM from this work \\
\hline SW3 (147) & $05: 35: 13.916$ & $-5: 22: 23.93$ & $-31.8 \pm 4.1$ & $10.1 \pm 4.6$ & $63.2 \pm 12$ & $288 \pm 15$ & PM from this work \\
\hline SW4 (6 cm blob) & $05: 35: 13.751$ & $-5: 22: 25.81$ & $\ldots$ & $\ldots$ & $\ldots$ & $\ldots$ & \\
\hline E1 & $05: 35: 14.532$ & $-5: 22: 20.71$ & $-141.1 \pm 48$ & $96.5 \pm 35$ & $324 \pm 113$ & $304 \pm 30$ & PM from this work \\
\hline E2 (“IRc 23”) & $05: 35: 14.609$ & $-5: 22: 21.02$ & $3.36 \pm 0.4$ & $20.1 \pm 8.6$ & $38.7 \pm 16$ & $9 \pm 15$ & PM from Dzib et al. (2017) \\
\hline E2 & $05: 35: 14.612$ & $-5: 22: 20.91$ & $12.62 \pm 2.7$ & $21.2 \pm 3.7$ & $46.7 \pm 9$ & $31 \pm 20$ & PM from this work \\
\hline
\end{tabular}

Note. All motions are in the Orion reference frame measured with respect to the mean proper motion (PM) of the radio positions of low-PM sources measured with the VLA in $C$ band at 4-8 GHz. The absolute PM of the Orion reference frame moves with $\mu_{\alpha} \cos \delta=1.07 \pm 0.09 \mathrm{mas} \mathrm{yr}^{-1}, \mu_{\delta}=-0.84 \pm 0.16 \mathrm{mas}^{\mathrm{yr}} \mathrm{r}^{-1}$ (Dzib et al. 2017). The absolute PMs reported by Goddi et al. (2011) have been transformed to the Dzib et al. (2017) OMC1 reference frame. A distance of $D=400$ pc is assumed for the conversion of PMs in the OMC1 frame to velocity.

the system of stars moves with velocity

$$
V_{\mathrm{CoM}}=\frac{\left(M_{\mathrm{BN}} V_{\mathrm{BN}}+M_{I} V_{I}+M_{x} V_{x}\right)}{\left(M_{\mathrm{BN}}+M_{I}+M_{x}\right)} .
$$

The masses of BN, Src $\mathrm{x}$, and Src I are allowed to span a slightly wider range than indicated above. The proper motions are allowed to vary in both amplitude and position angle over the range of uncertainties.

It is assumed that OMC1 is stationary in the reference frame defined by stars in the Orion Nebula Cluster. Furthermore, it is assumed that before the dynamical interaction ejected these runaway stars from $\mathrm{OMC} 1$, their $\mathrm{CoM}$ was moving through OMC1 with a speed less than the escape speed. We use the 
Table 3

Parameters for the Ejected Stars in the OMC1 Reference Frame

\begin{tabular}{lcccc}
\hline \hline Quantity & Src I & BN & Src x & Unit \\
\hline$V_{\text {PM }}$ & 9.4 & 25.6 & 55.1 & $\mathrm{~km} \mathrm{~s}^{-1}$ \\
$M$ & 15 & 12 & 3 & $M_{\odot}$ \\
$V_{r}$ & -4 & 14.2 & -32 & $\mathrm{~km} \mathrm{~s}^{-1}$ \\
$V_{\text {tot }}$ & 10.2 & 29.3 & 60 & $\mathrm{~km} \mathrm{~s}^{-1}$ \\
K.E. & 1.6 & 7.8 & 91 & $\times 10^{46} \mathrm{erg}$ \\
\hline
\end{tabular}

$850 \mu \mathrm{m}$ dust continuum map of Orion A from Lane et al. (2016) to estimate the mass of OMC1 as a function of radius from its center using Lane et al.'s (2016) Equation (1). The dust temperature as a function of distance from OMC1 is estimated by averaging the values from Dicker et al. (2009), De Buizer et al. (2012), and Chuss et al. (2019). The gas mass within $0.05 \mathrm{pc}$ of the ejection center is $M_{\mathrm{OMC} 1} \approx 100 M_{\odot}$ for a mean dust temperature $T_{d}=60 \mathrm{~K}$. The mass inside a circle with a radius of $0.5 \mathrm{pc}$ increases to $\sim 1300 M_{\odot}$ (for $T_{d}=30 \mathrm{~K}$ ). At these radii and assuming that the total enclosed mass at a given radius is dominated by the gas mass, the equivalent circular orbit speeds would be $V_{\text {circ }} \sim 2.9$ and $3.3 \mathrm{~km} \mathrm{~s}^{-1}$ at radii of 0.05 and $0.5 \mathrm{pc}$, respectively. The mass distribution is roughly that of an isothermal sphere with a relatively constant equivalent circular orbit speed. If the mean velocity of each star prior to ejection was equal to the equivalent circular orbit speed, the CoM motion is likely to be lower than the circular orbit speed by $\sqrt{3}$. Thus, the motion of the CoM is likely to be less than $\left\langle V_{\text {circ }}\right\rangle / \sqrt{3} \approx 1.9 \mathrm{~km} \mathrm{~s}^{-1}$.

Figure 3 show the calculated motion of the CoM in the Orion reference frame as functions of the stellar masses and proper motions. Each panel corresponds to a specific choice for the mass of Src x, starting with $M_{x}=1 M_{\odot}$ in the upper left. The mass is increased by $1 M_{\odot}$ in each panel and ends with $M_{x}=4 M_{\odot}$ in the lower right. For each choice of $M_{x}$, the mass of $\mathrm{BN}$ is allowed to vary from $M_{\mathrm{BN}}=8$ to $18 M_{\odot}$ to span the outer bounds of likely values; each choice of $M_{\mathrm{BN}}$ is given a unique color shown in the legend. For each choice of $M_{x}$ and $M_{\mathrm{BN}}$, the mass of Src I is allowed to vary from $M_{I}=5$ to $25 M_{\odot}$ in increments of $1 M_{\odot}$ to bracket the full range of plausible masses. $\left(M_{I}=5 M_{\odot}\right.$ corresponds to the top of each string of points while $M_{I}=25 M_{\odot}$ corresponds to the bottom).

The total mass of the three ejected stars is likely to be larger than $\sim 20 M_{\odot}$. Thus, because of equipartition of kinetic energy, their CoM is likely to have a motion with respect to the OMC1 frame that is considerably lower than the velocity dispersion of the majority of low-mass stars in the Orion Nebula Cluster (ONC). The blue, red, and yellow circles in Figure 3 indicate the speed of the CoM with respect to the ONC reference frame in steps of 1,2 , and $3 \mathrm{~km} \mathrm{~s}^{-1}$. The center of these circles is assumed to be at rest in the Orion Nebula reference frame determined from the mean motion of 79 radio stars, which defines a reference frame with $\mu_{\alpha} \cos (\delta)=1.07 \pm$ 0.09 mas yr $^{-1}$ and $\mu_{\delta}=-0.84 \pm 0.16$ mas yr $^{-1}$ (Dzib et al. 2017). At a distance $D=400 \mathrm{pc}, 1 \mathrm{mas} \mathrm{yr}^{-1}$ corresponds to motions of $1.896 \mathrm{~km} \mathrm{~s}^{-1}$, implying that the velocity dispersion

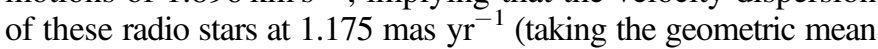
of the dispersion in R.A. and decl. from Dzib et al. 2017) is $\sim 2.2 \mathrm{~km} \mathrm{~s}^{-1}$, slightly larger than the blue circle in Figure 3.

A low mass for Src $\mathrm{x}$ shifts the CoM to the northwest. As the mass of Src $\mathrm{x}$ increases, the CoM shifts to the southeast for all likely Src I and BN masses. Figure 3 shows that in order for the
CoM of the Src I-BN-Src $\mathrm{x}$ system to move with a speed less than $\sim 2 \mathrm{~km} \mathrm{~s}^{-1}$ in the ONC reference frame, a low mass $\left(\sim 1 M_{\odot}\right)$ for Src $\mathrm{x}$ requires a high mass $\left(>11 M_{\odot}\right)$ for Src I, regardless of the mass of BN. On the other hand, a high mass for $\mathrm{Src} \mathrm{x}$ requires a relatively high mass $\mathrm{BN}\left(>10 M_{\odot}\right)$.

For the most likely mass of Src x, 2-3 $M_{\odot}$, a low mass for $\mathrm{BN}$ allows a low mass for Src I. For a more massive BN, the motion of the CoM tends to be away from the Trapezium toward the northwest. Thus, a higher Src I mass is required to keep the CoM motion at rest with respect to OMC1. For $M_{x}=3 M_{\odot}, M_{\mathrm{BN}}=12 M_{\odot}$ and $M_{I}>14 M_{\odot}$ if the $\mathrm{CoM}$ is moving away from the ONC with less than $1 \mathrm{~km} \mathrm{~s}^{-1}$. If the $\mathrm{CoM}$ of these ejected stars is at rest or falling toward the $\mathrm{ONC}$, a higher mass for Src I is more likely.

Variations of the proper-motion speeds and position angles over the range allowed for by the uncertainties listed in Table 1 were used to study their impact on the estimated motion of the CoM of the three stars. Varying the velocity and position angle of each star over the full range of allowed values while holding the corresponding parameters at their central values results in an error circle for the CoM motion. The radius of this error circle is about $0.4 \mathrm{~km} \mathrm{~s}^{-1}$, nearly independent over the range of stellar masses. Allowing all three speeds and position angles to vary over their full range of acceptable values simultaneously results in an error circle with a radius of about $0.6 \mathrm{~km} \mathrm{~s}^{-1}$, again relatively constant over the full range of stellar masses under consideration. As shown by Figure 3, the largest uncertainty is caused by the relatively poorly constrained mass of Src x.

\subsection{Constraints on the Radial Velocity of Src $x$}

Although the radial velocity of Src $\mathrm{x}$ has not been measured, it can be estimated from momentum conservation combined with the radial velocities and masses of BN and Src I from

$$
V_{r}(x)=-\frac{M_{I} V_{r}(I)+M_{\mathrm{BN}} V_{r}(\mathrm{BN})}{M_{x}} .
$$

Assuming that $V_{r}(\mathrm{BN})=+14.2 \pm 0.5 \mathrm{~km} \mathrm{~s}^{-1}$ and $V_{r}(I)=$ $-4 \pm 0.5 \mathrm{~km} \mathrm{~s}^{-1}$ in the OMC1 reference frame, the mass of Src $\mathrm{x}$ of $M_{x}=3 \pm 0.5 M_{\odot}, M_{\mathrm{BN}}=12 \pm 2 M_{\odot}$, and $M_{I}=$ $15 \pm 2 M_{\odot}$ imply $V_{r}(x)=-37 \pm 10 \mathrm{~km} \mathrm{~s}^{-1}$. Thus, the LSR radial velocity of $\operatorname{Src} \mathrm{x}$ is predicted to be $V_{\mathrm{LSR}}=-28 \pm$ $10 \mathrm{~km} \mathrm{~s}^{-1}$.

The study presented here differs from that of Farias \& Tan (2018), who also used the motion of the CoM of the ejected stars in the Orion frame to constrain the stellar masses in several respects. Our study explores a wider range of masses for Src x. We use the measured radial velocities of OMC1, BN, and Src I to constrain the likely radial velocity of Src x. Our analysis is broadly consistent with the conclusions of Farias \& Tan (2018). We present a graphical illustration of how this constraint implies a mass for Src I of around $15 M_{\odot}$, consistent with the direct mass measurement presented by Ginsburg et al. (2018).

Prior to the discovery of the large proper motion of Src $\mathrm{x}$ by Luhman et al. (2017), Src $n$ was though to be a runaway star ejected $550 \mathrm{yr}$ ago. As noted by Bally et al. (2017), it is not possible to reconcile the stellar proper motions with the requirement that the stellar CoM be stationary in the ONC reference frame. Indeed, the motions seemed to suggest that $\mathrm{CoM}$ and $\mathrm{OMC1}$ might be moving with a velocity of a few 


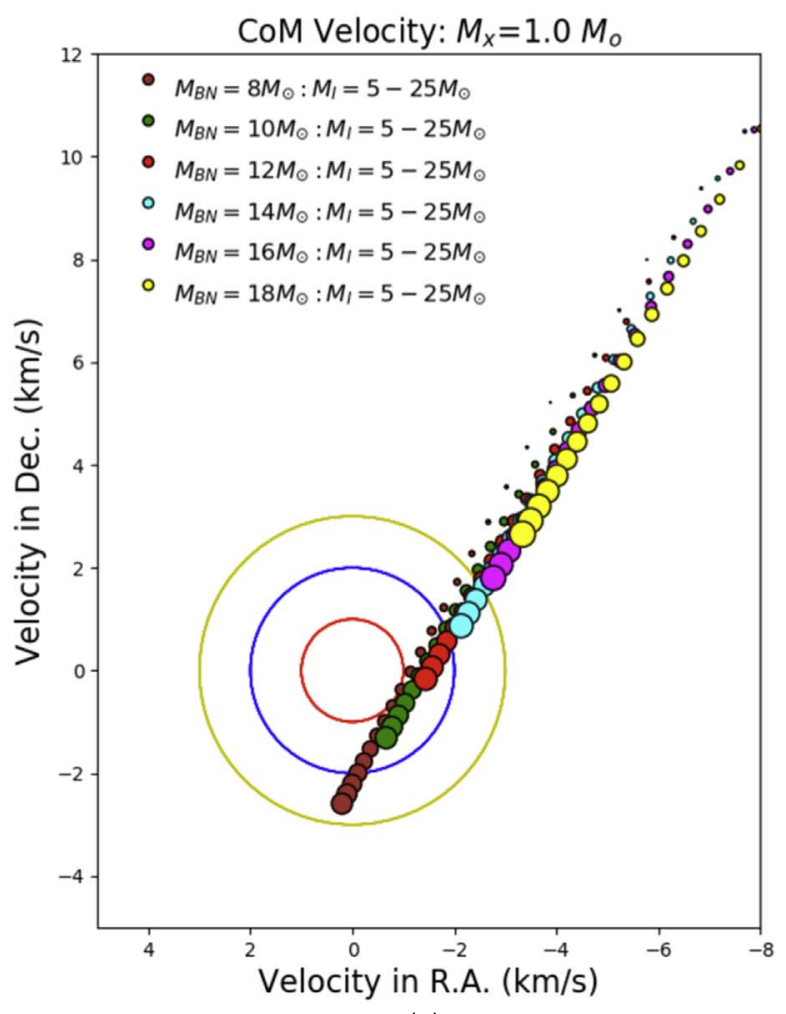

(a)

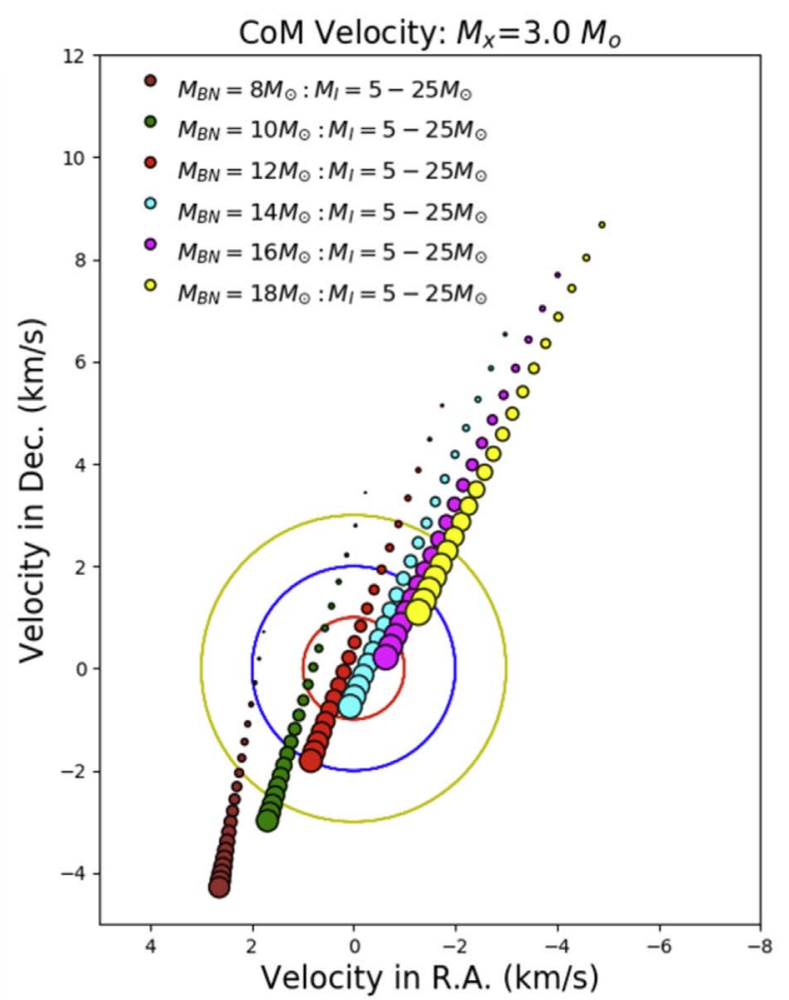

(c)

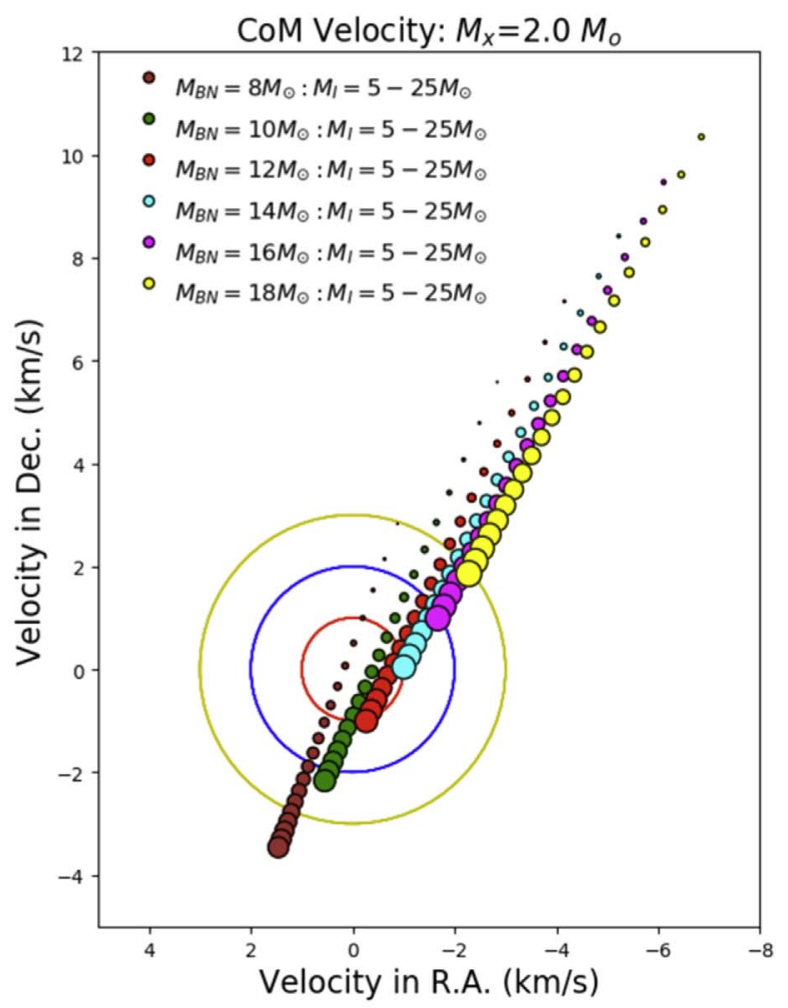

(b)

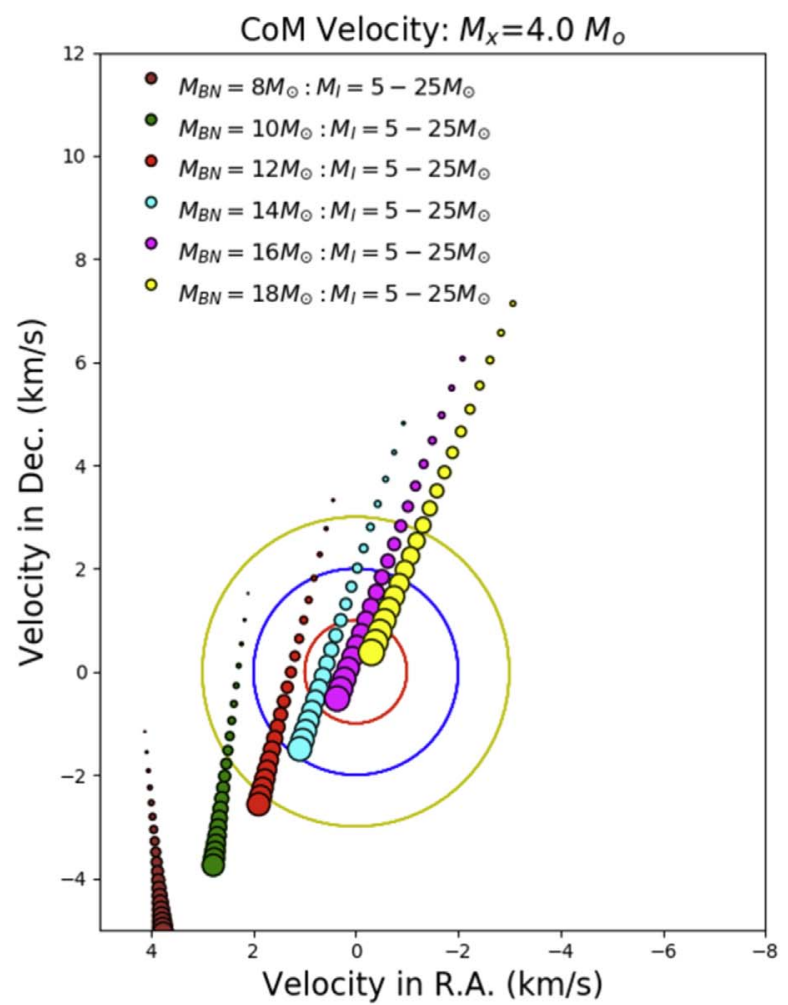

(d)

Figure 3. The velocity of the center of mass (CoM) of BN, Src I, and Src $\mathrm{x}$ as a function of the masses of Src $\mathrm{x}$, BN, and Src I. The position angles and proper motions assumed in this calculation are the best-fit values from Rodríguez et al. (2017) and Luhman et al. (2017) and assume that IRc 23 and Zapata 11 are very low-mass ejecta from BN. The red, blue, and yellow circles correspond to escape speeds of 1,2, and $3 \mathrm{~km} \mathrm{~s}^{-1}$ from OMC1 with respect to the CoM of the Orion Nebula Cluster. Each panel shows a specific choice for the mass of Src x. For each chosen mass of Src x, each panel shows the range of possible masses for BN shown in a unique color. For each Src x and BN mass, the mass of Src I varies from $5 M_{\odot}$ (top right) to $25 M_{\odot}$ (lower left). The circle diameters scale with the total mass, $M_{I}$ plus $M_{\mathrm{BN}}$. 


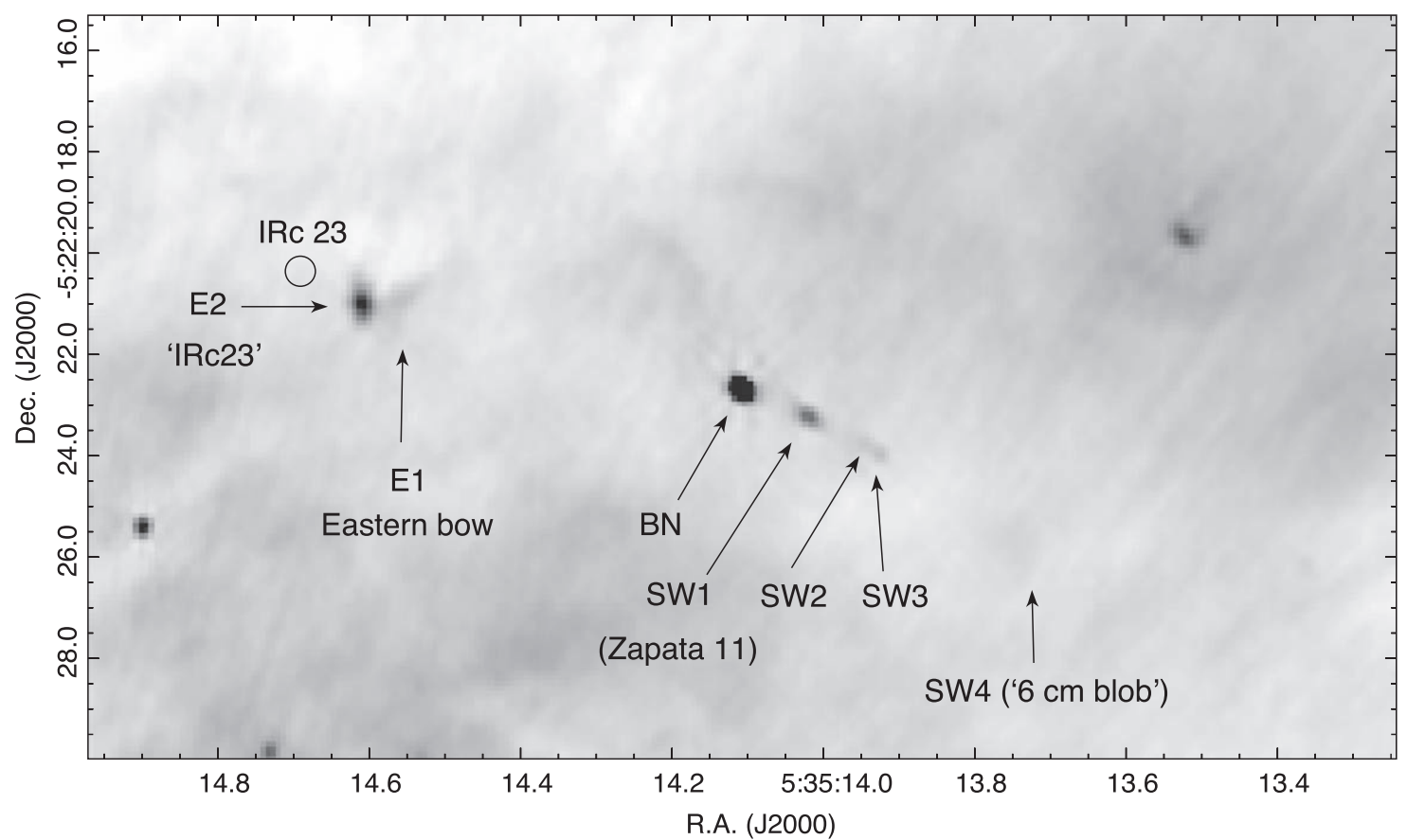

Figure 4. A close-up showing a small portion of the $6 \mathrm{GHz}$ radio continuum image from Forbrich et al. (2016) with the various objects listed in Table 4 indicated.

kilometers per second toward the west with respect to the ONC. The discovery that $\mathrm{Src} \mathrm{n}$ is not moving, but $\mathrm{Src} \mathrm{x}$ is, resolves this dilemma. Reasonable choices for the masses of the stars allows for no proper motion in the ONC frame.

It is possible that the mutual gravitational attraction between the $\mathrm{ONC}$ and $\mathrm{OMC} 1$ actually results in a net proper motion of $\mathrm{OMC1}$ and the CoM toward the southeast where the CoM of the ONC cluster lies (very close to the Trapezium stars, located $\sim 90^{\prime \prime}$ from OMC1). As shown in Figure 3, motion of the CoM and OMC1 toward Trapezium would favor a higher mass for both Src I and BN. This point was also discussed by Farias \& Tan (2018).

\subsection{A Slow Radio Jet from BN?}

Figure 4 shows a close-up of the region around $\mathrm{BN}$ in the 2012 epoch JVLA $6 \mathrm{GHz}$ data from Forbrich et al. (2016). BN is surrounded by a chain of diffuse radio sources consisting of SW4 $(6 \mathrm{~cm}$ blob), SW3, SW2, and SW1 (Zapata 11 in Dzib et al. 2017) located southwest of BN and E1 and E2 ("IRc 23" in Dzib et al. 2017) located east of BN. These objects trace a gently curving chain bending back toward the explosion center. The designations of these features in Forbrich et al. (2016) are indicated in Figure 5. We consider three possible interpretations for these radio features near BN.

First, as suggested by Dzib et al. (2017), they may contain lowluminosity protostars. If stars were embedded in the compact radio sources, the mass moving toward the north and northwest is larger than just BN. In this case, the mass of Src I needs to be increased to keep the CoM less than the escape speed from OMC1. However, there is no evidence for X-ray, infrared, or submillimeter counterparts to any of these radio features.

Second, given the apparent motion of at least two of the features, Zapata 11 and "IRc 23" (Dzib et al. 2017), away from the explosion center and the point from which BN, Src I, and Src $\mathrm{x}$ were ejected, the radio features may trace clumps ejected during the dynamic interaction and explosion $\sim 550 \mathrm{yr}$ ago. Their apparent association with BN is either a coincidence or indicates that they are clumps that became unbound from BN during its acceleration.

Third, they may trace a low-velocity outflow from BN. SW1 (Zapata 11; Zapata et al. 2004) and E2 ("IRc 23;" Dzib et al. 2017) exhibit proper motions away from the explosion center as well as away from BN (Figure 5). These objects are spatially extended in the $6 \mathrm{GHz}$ radio continuum. E2 is the brightest knot in the chain around $\mathrm{BN}$. New radio proper motions are presented below and listed in Table 2.

The radio source dubbed "IRc 23" by Dzib et al. (2017) but designated E2 here is offset by more than $1^{\prime \prime}$ from the mid-IR source IRc 23 first identified on $12.5 \mu \mathrm{m}$ images (Shuping et al. 2004). At $11.7 \mu \mathrm{m}$ (Smith et al. 2005), IRc 23 is a condensation along the eastern edge of the mid-IR nebula centered on BN that abuts the western edge of a cometary dust cloud seen at millimeter wavelengths (Figure 5).

A "disk shadow" (Figure 5) and bipolar reflection nebula is centered on IRc 23 at the southwest-facing head of the cometary cloud. This nebula indicates the presence of a star embedded in a nearly edge-on disk with an axis at $\mathrm{PA} \sim 105^{\circ} \pm 5^{\circ}$ (disk shadow is elongated toward PA $=\sim 15^{\circ}$ ). A 16th magnitude star (Vega scale) is visible at the center of the "disk shadow" in the $K_{s}$ adaptive optics images (Bally et al. 2015). No compact 1.3-3 mm source brighter than $0.1 \mathrm{mJy} /$ beam is seen in the 0. . 03 resolution ALMA continuum images associated with data presented by Ginsburg et al. (2018, 2019).

E2 (designated \#68 in Forbrich et al. 2016 and "IRc 23" in Dzib et al. 2017) is an arcsecond-scale $6 \mathrm{GHz}$ knot elongated north to south about 1!" 3 southwest of IRc 23 (Figure 5). A dim, 2" long, crescent-shaped radio feature, E1, is located about 0 " 5 west of E2. E1 resembles a bow shock moving due east from BN. E2 and E1 may trace an outflow running into the western rim of the cometary dust cloud.

SW1 (designated \#157 in Forbrich et al. 2016 and Zapata 11 in Dzib et al. 2017) is the brightest knot in a jet-like chain of radio continuum emission extending southwest from $\mathrm{BN}$ containing 


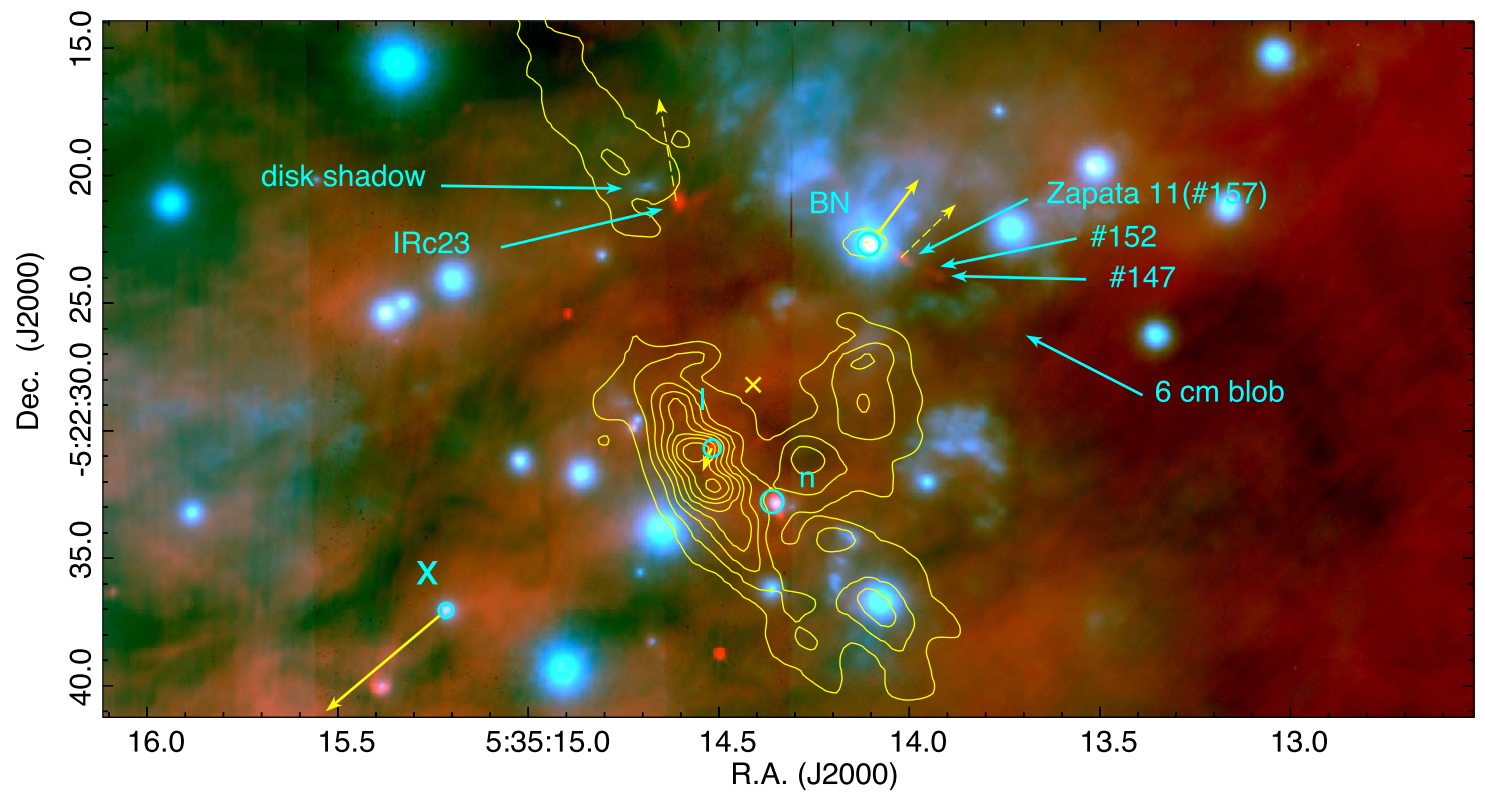

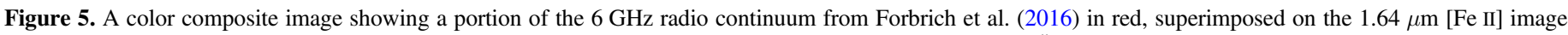

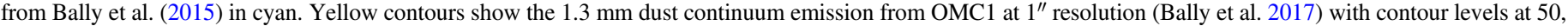

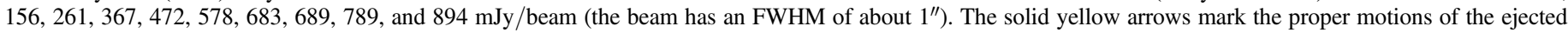

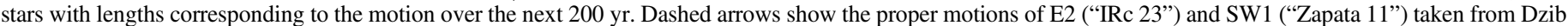

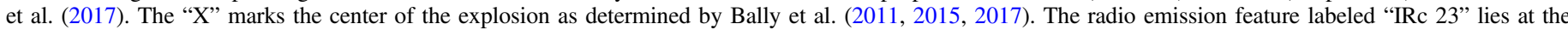

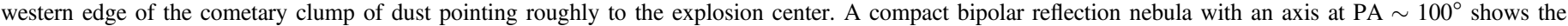

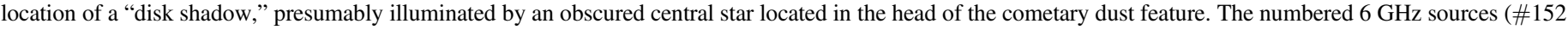

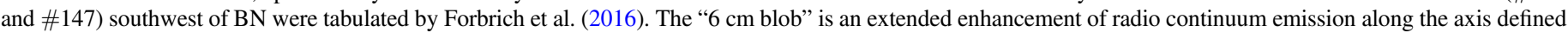
by Zapata $11, \# 152$, and \#147.

SW2 and SW3 (Forbrich et al. 2016 objects \#152, \#147), which terminates in a very dim blob, SW4 ("6 $\mathrm{cm}$ blob" in Figure 5).

None of these radio features contain compact $1.3 \mathrm{~mm}$ sources in the ALMA continuum image. The $1.3 \mathrm{~mm}$ flux limit is $\sim 0.1 \mathrm{mJy}$ in a $0 .{ }^{\prime \prime} 03$ beam. In the $11.7 \mu \mathrm{m}$ image of Smith et al. (2005), there are no compact counterparts brighter than $0.3 \mathrm{Jy}$ at the locations of E1 and E2, SW1, SW2, SW3, or SW4. However, there is a local intensity maximum near SW1. In the $12.5 \mu \mathrm{m}$ image (Shuping et al. 2004), this feature is labeled "SW Arc" and appears to be part of the bright mid-IR nebulosity around BN. In the $K_{s}$ adaptive optics image from Bally et al. (2015), there are no compact $(<0$ !' 1$)$ sources brighter than magnitude 18 at the locations of the $6 \mathrm{GHz}$ knots. However, bright diffuse emission due to reflection nebulosity and $\mathrm{H}_{2}$ emission limits sensitivity. Grosso et al. (2005) used the Chandra X-ray Observatory to observe OMC1 between 0.5 and $8 \mathrm{keV}$ for an on-source exposure of $838 \mathrm{ks}$ (9.7 days). There are no compact X-ray sources at the locations of these radio features. The limits on the X-ray luminosity are $L_{\mathrm{x}}<10^{27} \mathrm{erg} \mathrm{s}^{-1}$.

Assuming that the string of knots near BN traces an outflow, the mean position angle of this flow on the plane of the sky is PA $\sim 69^{\circ}$. Multiple knots in a chain indicate that the outflow is collimated into a velocity-variable beam with a width of hundreds of astronomical units.

\subsection{New Proper Motions and Variations of the $6 \mathrm{GHz}$ Features near $B N$}

We use the 2012 and 2016 VLA data to measure new proper motions and to identify variations in the radio emission. ${ }^{4}$ Table 2 lists these measurements; Table 4 gives these proper motions

\footnotetext{
4 A full discussion of proper motions derived from a comparison of these two data sets will appear in J. Vargas-González et al. (2020, in preparation).
}

relative to the motion of BN. SW1, SW3, and E2 are moving away from BN with speeds of $28 \pm 9$ to $45 \pm 20 \mathrm{~km} \mathrm{~s}^{-1}$. SW3 brightened from a peak flux of $\sim 80 \mu \mathrm{Jy}$ in 2012 to $\sim 300 \mu \mathrm{Jy}$ in 2016 and SW2 increased from $\sim 60$ to $\sim 300 \mu \mathrm{Jy}$. Additionally, the structure of knots SW2 and E1 changed significantly over the $4 \mathrm{yr}$ interval. The photocenters of these features seem to be moving back toward $\mathrm{BN}$ ! A possible interpretation is that SW2 on the 2012 image merged with SW3 by 2016, resulting in the more than factor of 3 increase in the flux of SW3. Additionally, a new knot has appeared in 2016 slightly east of where SW2 was located in 2012. The diffuse feature SW4 was not recovered in the shallower 2016 epoch images.

The photocenter of the northwest edge of E1, the "eastern bow" located $\sim 1^{\prime \prime}$ west of E2, shifted toward the northwest. Fitting the photocenters gives an apparent proper motion of $\sim 300 \mathrm{~km} \mathrm{~s}^{-1}$. However, it is more likely that this "motion" is caused by intensity variations: fading of one part of the "eastern bow" combined with the brightening of another part. Given the errors, the motion of $\mathrm{BN}$ is consistent with previously measured proper motions using longer time intervals.

\subsection{Electron Densities of the $6 \mathrm{GHz}$ Features near BN}

Table 5 lists the background-subtracted, peak and areaintegrated flux densities, the radii of the regions in which the flux densities were measured, the projected distance from BN, and the estimated mean electron density in each knot in Figure 4 measured on the 2012 epoch $6 \mathrm{GHz}$ image. The spectral index of the knots between 4.7 and $7.3 \mathrm{GHz}$ is flat. Thus, we assume that the radio continuum emission is produced by optically thin bremsstrahlung.

The optical depth of free-free emission is given by $\tau=3.28 \times 10^{-7} T_{4}^{-1.35} \nu_{1}^{-2.1} \mathrm{EM}$ (Condon \& Ransom 2016), 
Table 4

Proper Motions ${ }^{\mathrm{a}}$ of Suspected BN Outflow Features Relative to BN

\begin{tabular}{|c|c|c|c|c|c|}
\hline Object & $\begin{array}{c}\mu_{\alpha} \cos (\delta) \\
\left(\mathrm{mas}^{-1}\right)\end{array}$ & 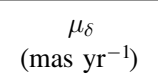 & $\begin{array}{c}V_{t} \\
\left(\mathrm{~km} \mathrm{~s}^{-1}\right)\end{array}$ & $\begin{array}{l}\text { P.A. } \\
\text { (deg) }\end{array}$ & Comments \\
\hline SW1 & $-14.0 \pm 9.4$ & $-5.4 \pm 5.4$ & 28.4 & 248 & Close to $\mathrm{BN}$ \\
\hline SW2 & $25.3 \pm 9.8$ & $-19.6 \pm 6.2$ & 60.7 & 128 & New component in $2016 ?$ \\
\hline $\mathrm{E} 1^{\mathrm{b}}$ & $-133 \pm 50$ & $85.7 \pm 35$ & 300 & 303 & Bow with photometric variations \\
\hline E2 & $20.7 \pm 3.0$ & $10.4 \pm 4.0$ & 43.9 & 63 & Bright knot near IRc 23 \\
\hline
\end{tabular}

Notes.

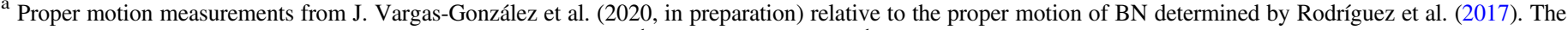

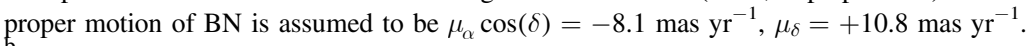

${ }^{\mathrm{b}}$ Source not considered in the catalogs of Forbrich et al. (2016) or J. Vargas-González et al. (2020, in preparation; S/N < 5).

Table 5

Parameters of the BN Radio Jet

\begin{tabular}{|c|c|c|c|c|c|c|}
\hline Object & $\begin{array}{l}\text { Peak } S_{\nu} \\
(\mu \mathrm{Jy})\end{array}$ & $\begin{array}{c}\text { Total } S_{\nu} \\
(\mu \mathrm{Jy})\end{array}$ & $\begin{array}{l}\text { Size } \\
\left({ }^{\prime \prime}\right)\end{array}$ & $\begin{array}{c}D_{\mathrm{BN}} \\
\left({ }^{\prime \prime}\right)\end{array}$ & $\begin{array}{c}n_{e} \\
\left(\times 10^{4} \mathrm{~cm}^{-3}\right)\end{array}$ & Comments \\
\hline$\overline{\mathrm{SW} 1}$ & 270 & 416 & $0 . " 4 \times 0 . " 8$ & 1.5 & 5.2 & $157^{\mathrm{a}}(\text { Zapata } 11)^{\mathrm{b}}$ \\
\hline SW2 & 64 & 41 & $0 " 3$ & 2.7 & 1.9 & $152^{\mathrm{a}}$ \\
\hline SW3 & 80 & 50 & 0.3 & 3.1 & 2.1 & $147^{\mathrm{a}}$ \\
\hline SW4 & 25 & 260 & $1 " \prime 6$ & 6.3 & 2.0 & $6 \mathrm{~cm}$ blob \\
\hline $\mathrm{BN}$ & 2320 & 2473 & $0 " 6$ & $\cdots$ & $\cdots$ & \\
\hline E1 & 78 & 2650 & $0 . ! 3 \times 0 . ! 67$ & 6.9 & 15 & Bow near IRc 23 \\
\hline E2 & 47 & 1048 & $0 . .25 \times 0$ ". 40 & 7.7 & 10 & "IRc 23" \\
\hline
\end{tabular}

Notes.

a Designation in Forbrich et al. (2016).

${ }^{b}$ Designation in Dzib et al. (2017).

where $T_{4}$ is the plasma temperature in units of $10^{4} \mathrm{~K}, \nu_{1}$ is the frequency in units of $1 \mathrm{GHz}, \mathrm{EM}=\int_{0}^{L} n_{e}^{2}(x) d x \approx$ $n_{e}^{2} L\left(\mathrm{~cm}^{-6} \mathrm{pc}\right)$ is the emission measure in the usual units, $n_{e}$ is the electron density, and $L$ is the line-of-sight path length through the emission region (in units of parsec). Thus, $n_{e} \approx(\mathrm{EM} / L)^{1 / 2}\left(\mathrm{~cm}^{-3}\right)$. When $\tau \ll 1$, the flux density is given by $S_{\nu}=2 k T \nu^{2} \Omega_{B}(\mathrm{sr}) \tau / c^{2}$. Thus,

$$
S_{\nu}(\mathrm{Jy})=100.77 T_{4}^{-0.35} \nu_{1}^{-0.1} \Omega_{B}(\mathrm{sr}) \mathrm{EM} .
$$

For the JVLA $6 \mathrm{GHz}$ observations the synthesized beam diameter is approximately 0 !" 36 , implying that $\Omega_{B}=2.39 \times 10^{-12} \mathrm{sr}$. With $\nu=6.099 \mathrm{GHz}, \operatorname{EM}\left(\mathrm{cm}^{-6} \mathrm{pc}\right)=4971 S_{\nu}(\mu \mathrm{Jy})$, where the flux density is expressed in units of $\mu \mathrm{Jy}$. The mean electron density is then given by

$$
n_{e} \approx\left[\frac{4971 S_{\nu}(\mu \mathrm{Jy})}{L_{\mathrm{pc}}}\right]^{1 / 2}\left(\mathrm{~cm}^{-3}\right) .
$$

The electron densities given in Table 5 assume that for nonround features, the line-of-sight depth is comparable to the minor axis size of the region under consideration. Thus, the electron densities range from $10^{4}$ to about $10^{5} \mathrm{~cm}^{-3}$, about one to two orders of magnitude higher density than the mean electron density of the Orion Nebula. If these knots are only partially ionized, their hydrogen densities will be higher.

Based on the proper motion measurements, SW1, SW3, and E2 are moving away from BN (Table 4). The lower bound on the masses of the features range from $10^{-7} M_{\odot}$ to $10^{-5} M_{\odot}$. Their momenta are at least $10^{-5} M_{\odot} \mathrm{km} \mathrm{s}^{-1}$ to $3 \times 10^{-4} M_{\odot} \mathrm{km} \mathrm{s}^{-1}$; lower bounds on their kinetic energies in the $\mathrm{BN}$ frame are $10^{39}-10^{41} \mathrm{erg}$. For a mean speed of $35 \mathrm{~km} \mathrm{~s}^{-1}$, their dynamic ages, $\tau_{\text {dyn }}=R / V$, range from $\sim 80 \mathrm{yr}$ (SW1) to $400 \mathrm{yr}$ for $\mathrm{E} 2$.

\section{Discussion}

\subsection{Consequences for the Motion of the Center of Mass}

The lack of compact stellar sources in the $5 \mathrm{GHz}$ knots implies that they be ignored in the analysis of the motion of the CoM of ejected stars. However, if they did contain significant mass such as a brown dwarf or a star, then the mass of Src I must be increased to keep the motion of the CoM of the ejected objects smaller than the velocity dispersion of OMC1.

A somewhat greater uncertainty is introduced by the mass and momentum of the ejected gas in the OMC1 high-velocity outflow and explosion (Figure 6). However, as discussed in Bally et al. (2017), $1^{\prime \prime}$ resolution observations of the $\mathrm{CO}$ in the flow with ALMA show that to first order, the explosion in the gas is isotropic. To second order, the flow is elongated along a southeast-northwest axis with a slight deflection of the southeastern lobe toward the east. These deviations from perfect symmetry are evident in Figures 1 and 6. Because the flow is roughly elongated in a direction parallel to the proper motions of BN and Src I, this asymmetry is likely to only have a minor impact on the motion of the CoM of the ejected stars.

\subsection{Constraints on the BN Disk}

Near-IR $1.6 \mu \mathrm{m}$ images of $\mathrm{BN}$ in polarized light reveal a bipolar reflection nebula with an axis of symmetry at $\mathrm{PA} \approx 36^{\circ}$ (Jiang et al. 2005). The dark lane in the nebula is close to BN's 


\title{
Max. Temperature
}

\author{
$\mathrm{V}_{\text {LSR }}=-100$ to $0 \mathrm{~km} / \mathrm{s}$ \\ +20 to +120 km/s
}


emission as hot $\mathrm{CO}(T \sim 3000-5000 \mathrm{~K})$ excited by collisions with $\mathrm{H}_{2}$ in a high-density environment $\left(n\left(\mathrm{H}_{2}\right)>10^{10} \mathrm{~cm}^{-3}\right)$ within $\sim 1$ au of BN.

Indriolo et al. (2018) obtained sensitive mid-IR spectra around $6 \mu \mathrm{m}$ toward $\mathrm{BN}$ and failed to detect either $\mathrm{H}_{2} \mathrm{O}$ or $\mathrm{CO}$ in absorption toward the star near its radial velocity of $\sim 20 \mathrm{~km} \mathrm{~s}^{-1}$. The absence of absorption toward $\mathrm{BN}$ at these velocities implies that the disk seen in emission in the vibrationally excited lines of $\mathrm{CO}$ cannot be edge-on. The near-IR bipolar nebula, combined with the $\mathrm{CO}$ vibrational band results, implies a significant tilt of the disk axis with respect to the line of sight so that most of the extinction and absorption toward $\mathrm{BN}$ must arise from the foreground cloud between $\mathrm{BN}$ and the Orion Nebula ionization front.

\subsection{The Outflow from $B N$}

$\mathrm{BN}$ is moving through the molecular cloud behind the Orion Nebula between the dense filaments and cores traced by the dust continuum (Figure 5). Thus, the compact $6 \mathrm{GHz}$ radio features are likely to be propagating into this interfilament molecular gas whose density is poorly constrained. Assuming that $\mathrm{BN}$ is $0.1-0.2$ pc behind the Orion Nebula's ionization front and using an extinction of $A_{V} \sim 25 \mathrm{mag}$ toward $\mathrm{BN}$ (Scoville et al. 1983) implies a mean density along the line of sight to BN of $n\left(\mathrm{H}_{2}\right) \sim 3-6 \times 10^{4} \mathrm{~cm}^{-3}$, the same order of magnitude as the electron densities of the radio knots.

What ionizes the knots? Observations of the free-free and $\mathrm{H}$-recombination line emission from $\mathrm{BN}$ indicates that ionizing Lyman continuum radiation from BN is confined to within $20 \mathrm{au}$. Thus, ionization of these radio features is likely caused by strong shocks. To ionize hydrogen, the shocks must be fast enough to either produce Lyman continuum emission which ionizes the preshock medium in a radiative precursor, or be sufficiently hot to collisionally ionize hydrogen in the postshock layer. Dissociation of $\mathrm{H}_{2}$ into $\mathrm{HI}$ followed by ionization of at least $50 \%$ of $\mathrm{HI}$ requires a postshock temperature of $T_{\mathrm{ps}} \sim \Phi / 10 \mathrm{k}>15,000 \mathrm{~K}$ where $\Phi=13.6 \mathrm{eV}$ is the ionization potential of H I (Draine 2011).

Thus, significant collisional ionization of hydrogen requires a shock speed $V_{\mathrm{ps}}>\left(16 \bar{n} k T_{\mathrm{ps}} / 3 \mu m_{\mathrm{H}}\right)^{1 / 2} \sim 30 \mathrm{~km} \mathrm{~s}^{-1}$. Here, $\mu \approx 2.4$ is the mean molecular weight in the preshock molecular cloud (assuming $Z=0.70, Y=0.28$, and $Z=0.02$ ), $\bar{n}$ is the number of particles in the postshock medium created by dissociation and ionization per incident $\mathrm{H}_{2}$ molecule (four particles when $\mathrm{H}_{2}$ is dissociated and fully ionized). For $50 \%$ ionization of $\mathrm{H}, \bar{n} \sim 3$. Given that the BN disk is likely to be inclined, the measured proper motions imply that the actual shock speeds are likely to be $\sim 30$ to $50 \mathrm{~km} \mathrm{~s}^{-1}$.

The recombination time for a plasma with electron density $n_{e}$ is $t_{\mathrm{rec}}=1 / n_{e} \alpha_{B}$. For $n_{e}=10^{4}-10^{5} \mathrm{~cm}^{-3}, t_{\mathrm{rec}}=1.2-12 \mathrm{yr}$. Thus, it is not surprising that large photometric variations are observed on a timescale of $\sim 4$ yr or that the sizes of the shocks in the $\mathrm{BN}$ outflow have subarcsecond dimensions along their minor axes.

High spectral resolution observations of the near-IR, hydrogen recombination lines provide evidence for a dense, ionized wind emanating from BN with $\dot{M} \sim 4 \times 10^{-7} M_{\odot} \mathrm{yr}^{-1}$ (Scoville et al. 1983). Beuther et al. (2010) presented higher signal-to-noise data confirming the broad H-recombination lines with an FWHM of $\sim 175 \mathrm{~km} \mathrm{~s}^{-1}$ along with a brighter compact line core with an FWHM of $\sim 39.3 \mathrm{~km} \mathrm{~s}^{-1}$. The wind remains ionized to a radius of $\sim 20$ au from BN, where it has a density $n_{e} \sim 1.3 \times 10^{7} \mathrm{~cm}^{-3}$.
Best fits to the Brackett $\alpha$ and $\gamma$ line profiles indicate a wind velocity profile that declines as $V_{w}=V_{o}\left(r / r_{0}\right)^{-2 / 3}$, where $r_{0}=$ $0.025 \mathrm{au}$ (Scoville et al. 1983). For an initial wind velocity of 1 to $2 \times 10^{3} \mathrm{~km} \mathrm{~s}^{-1}$ at the base of the wind, assumed to be located at the $r=0.025$ au stellar radius of a ZAMS B0 star, this velocity profile implies that the wind decelerates to $11-23 \mathrm{~km} \mathrm{~s}^{-1}$ at $r=20 \mathrm{au}$. However, slight deviations from the above powerlaw wind velocity profile can result in a higher wind speed. Proper motions and shock ionization of the knots indicate that the flow speed must be at least $30-50 \mathrm{~km} \mathrm{~s}^{-1}$ at distances of a few hundred astronomical units from BN. Our observations indicate that on larger scales, this wind is collimated into a velocityvariable jet emerging nearly orthogonal to BN's compact circumstellar disk and the star's proper motion.

\subsection{Dynamical Ejection and Disk Properties}

Src I is surrounded by a massive $\left(\sim 0.1 M_{\odot}\right)$, nearly edge-on, $\sim 50$ au radius disk whose spin axis is nearly orthogonal to the Src I proper motion vector (Hirota et al. 2017; Ginsburg et al. $2018,2019)$. The Src I spin axis is at PA $\approx 53^{\circ}$. The BN object is also surrounded by $<20$ au disk. Its spin axis is also nearly at right angles to the proper motion as indicated by its nearinfrared reflection nebula. Both Src I and BN have outflows younger than $500 \mathrm{yr}$ old ejected roughly at right angles to their proper motions.

Src $\mathrm{x}$ has near- to mid-IR excess, indicating a warm and likely compact circumstellar component. However, Src x is not detected at millimeter or submillimeter wavelengths. Thus, any disk associated with this source must have a much smaller mass than the disks surrounding Src I and BN.

Close periastron passages during the final $N$-body interaction leading to stellar ejection can severely perturb or disrupt preexisting circumstellar disks and envelopes. The abrupt acceleration during a close $N$-body encounter that imparts a velocity $V$ to a star will tend to strip off and eject circumstellar matter beyond the gravitational radius, $R_{G} \approx G M / V^{2}$, where $M$ is the mass of the accelerated star. On the other hand, material located inside $R_{G}$ will tend to be dragged along because it is sufficiently bound by the star's gravity (the Kepler speed is greater than the star's kick speed).

For Src I with $M_{I}=15 M_{\odot}$ and $V_{I}=10.2 \mathrm{~km} \mathrm{~s}^{-1}, R_{G} \approx$ $128 \mathrm{au}$. The measured radius of the Src I disk ranges from $R_{I}=37$ to 51 au (Ginsburg et al. 2018), well within the current gravitational radius of Src I. For BN with $M_{\mathrm{BN}}=12 M_{\odot}$ and $V_{\mathrm{BN}}=30 \mathrm{~km} \mathrm{~s}^{-1}, R_{G} \approx 12 \mathrm{au}$, similar to the observed radius of the submillimeter emission from BN. For Src x with $M_{x}=3 M_{\odot}$ and $V_{x}=55 \mathrm{~km} \mathrm{~s}^{-1}, R_{G} \approx 0.9 \mathrm{au}$, consistent with the lack of detection of a disk but the presence of bright near- to mid-IR emission.

Multiple encounters prior to stellar ejection can remove additional matter from a disk or envelope. A rough estimate of the fractional area of a disk impacted by a passing star with mass $M_{2}$ moving with speed $V_{2}$ in the potential of a more massive star with mass $M_{1}$ can be obtained from the gravitational radius of $M_{2}, R_{2}(r) \approx G M_{2} / V_{2}^{2}(r)$, where $r$ is the periastron separation when the intruder star plunges through the disk. Matter closer to the intruder than $R_{2}(r)$ is likely to be ejected from the disk around $M_{1}$ or become captured by the intruder. If the intruder also has a disk, the cross section for interaction can be larger.

Because the intruder star is most likely to have been born from the same clump as the primary, its speed during disk 


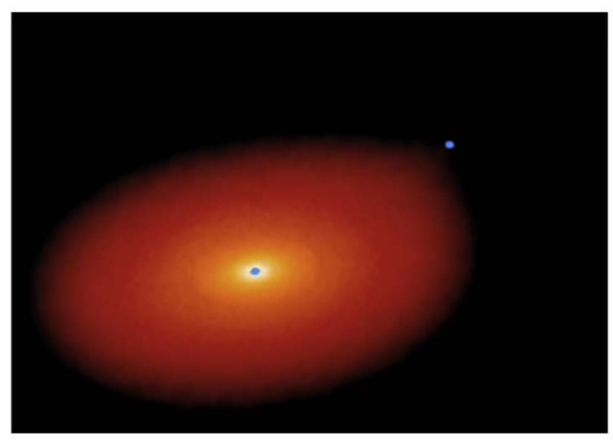

(a)

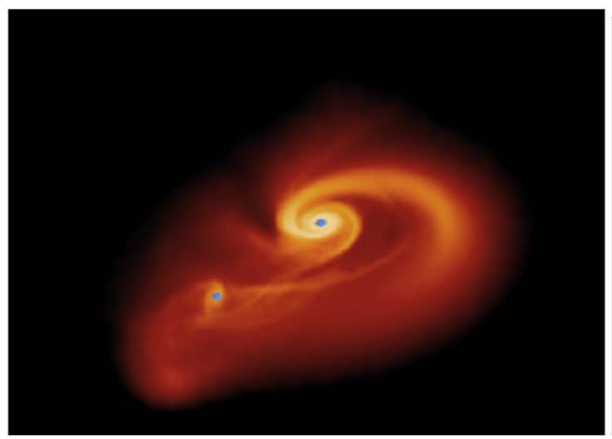

(d)

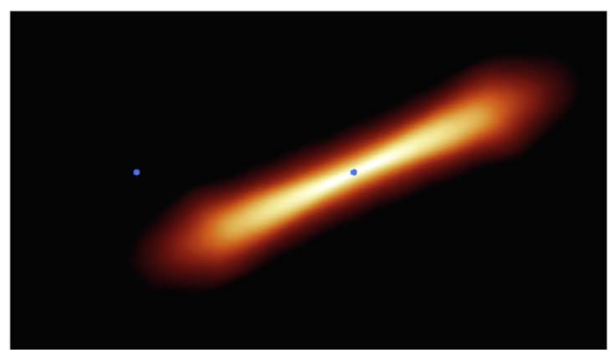

(g)

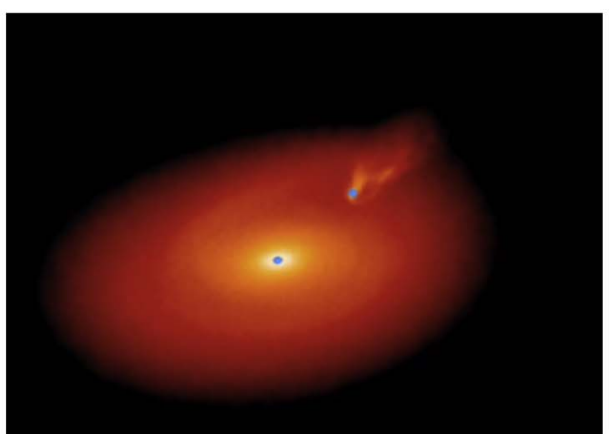

(b)

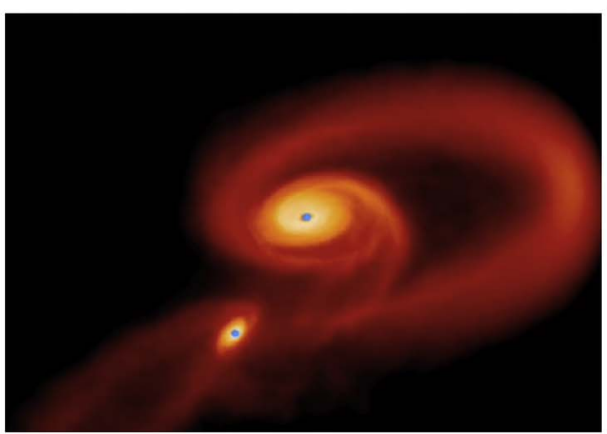

(e)

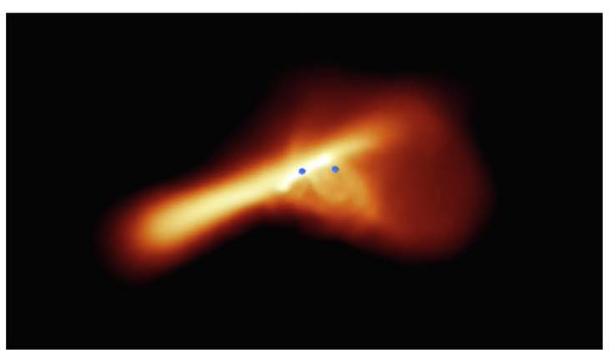

(h)

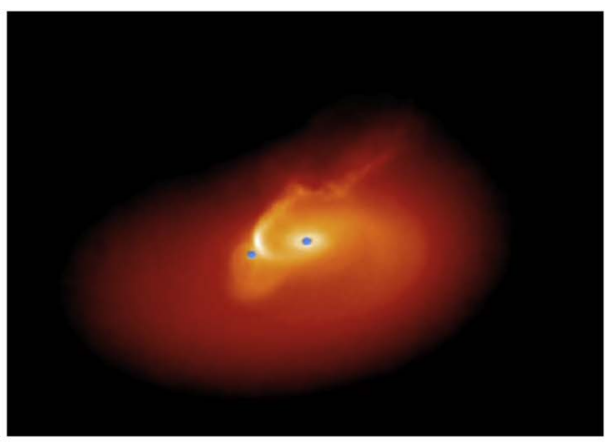

(c)

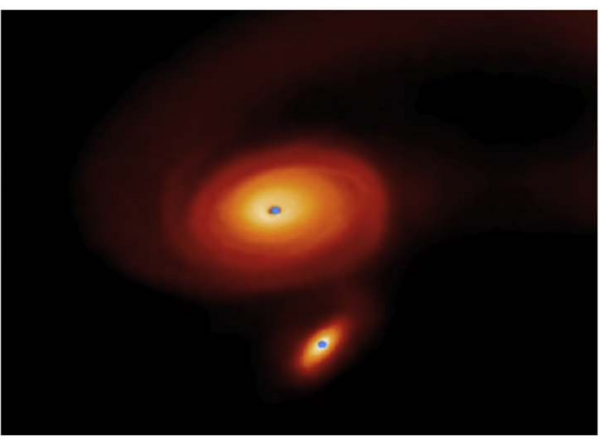

(f)

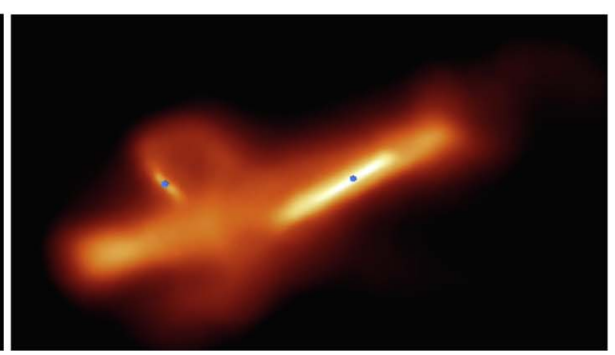

(i)

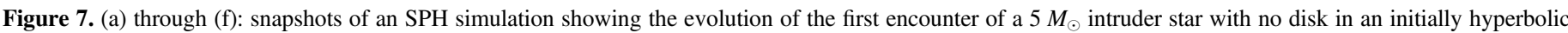

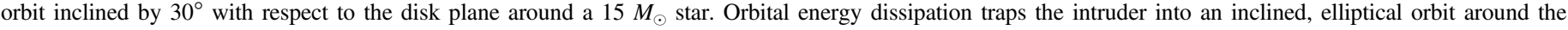

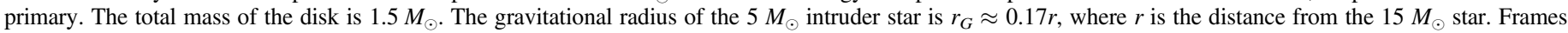

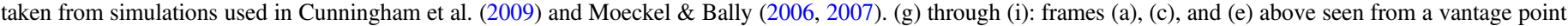
located to the right in frames (a) through (f) and lying in the initial disk plane of the massive star.

impact, $V_{2}(r)$, can be approximated by the escape speed at $r$ from the gravity well of the primary with mass $M_{1}$. (The intruder star is accelerated by the gravitational potential of the primary.) Thus, $r_{G} \approx r M_{2} / 2 M_{1}$.

If the disk outer radius is $r_{d}$, the fraction of the disk area impacted by an intruder impacting the disk at right angles is $f \approx\left(r_{G} / r_{d}\right)^{2}$, or

$$
f \approx \frac{1}{4}\left(\frac{r}{r_{d}}\right)^{2}\left(\frac{M_{2}}{M_{1}}\right)^{2}
$$

which is a lower bound. As the orbit plane of the intruder approached the disk plane, the impacted area increases because the area defined by $r_{G}$ becomes an ellipse with semimajor axis $a_{G} \approx r_{G} / \sin (\theta)$, where $\theta$ is the angle between the two planes. The most destructive encounter would be one in which the orbit plane of the secondary lies within the plane of the primary's disk. Multiple encounters prior to the final stellar ejection can progressively remove ever larger portions of the disk, leaving retained material in a highly perturbed state. Each time an intruder star passes through a disk, its likely to eject some mass.

The massive disk around Src I is likely to be the surviving remnant of a primordial disk that predated the dynamic ejection and explosion $\sim 500 \mathrm{yr}$ ago. If more than one star was surrounded by a disk, material could have been exchanged or caught by the object that became Src I. Similarly, parts of the Src I disk might have been trapped by the other ejected stars. The retained parts of the disks would have been highly perturbed and shocked. The event likely heated the entire disk (Ginsburg et al. 2019). However, the noncircular and noncoplanar motions would be damped by shocks within a few orbits. At a radius of $50 \mathrm{au}$, the orbit time around a $15 M_{\odot}$ Src I is $\sim 100 \mathrm{yr}$, much shorter than the $\sim 550 \mathrm{yr}$ since the ejection of Src I. Thus, it is not surprising that today, the Src I disk looks relaxed.

Figure 7 shows several frames from a simulation of a penetrating encounter (Moeckel \& Bally 2006, 2007; Cunningham et al. 2009). In this 3D smoothed particle hydrodynamics (SPH) simulation, a $15 M_{\odot}$ star is surrounded by a massive, $1.5 M_{\odot}$ disk 


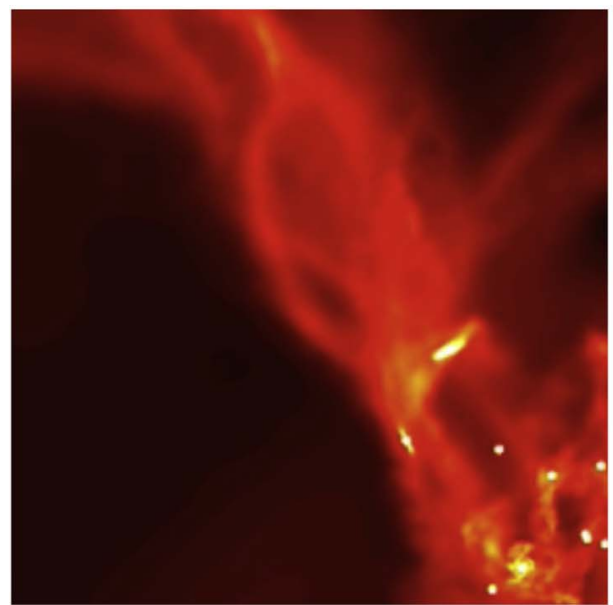

(a)

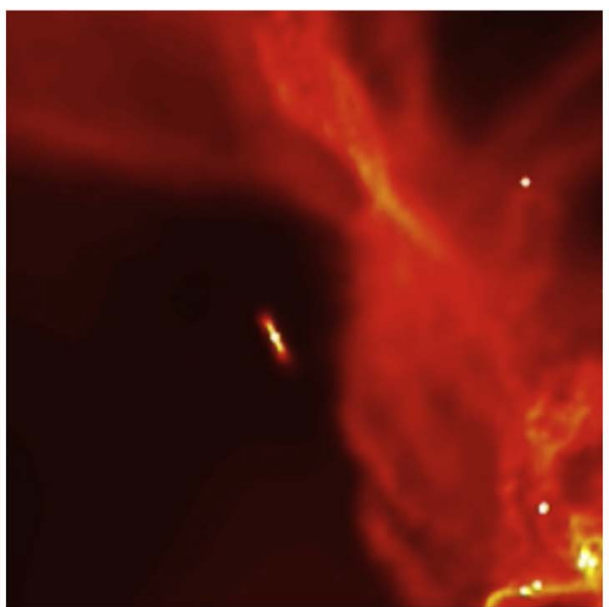

(b)

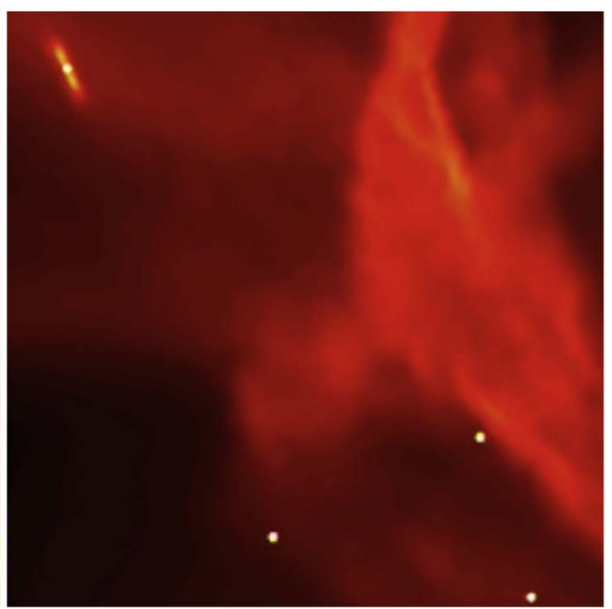

(c)

Figure 8. Three frames from an SPH simulation showing the final reorientation of a disk around an ejected star. Taken from Bate et al. (2002a, 2002b).

with a surface density declining as $r^{-1}$ and temperature declining as $r^{-1 / 2}$. A naked, $5 M_{\odot}$ star on a hyperbolic orbit with an orbit plane inclined $30^{\circ}$ with respect to the disk plane of the primary encounters the disk (Figure 7(a)). During early phases of the encounter, a region within the gravitational radius of the intruder is impacted (Figure 7(b)). As the intruder loops around the massive star, the disk develops transient spiral arms where gas is strongly shocked (Figures 7(c)-(e)). Parts of the outer disk are ejected ,and the intruder steals material to form its own disk with an outer radius roughly given by its gravitational radius near periastron (Figures 7(e) and (f)). Figures 7(g)-(i) show three frames of this encounter from the vantage point of an observer located in the plane of the initial disk around the massive star. The disks relax within a few orbit times at the disk outer radii.

The Src I and BN disks have spin axes roughly at right angles to their proper motions. Within the gravitational radius, abrupt acceleration of a star will reorient bound orbits. In the CoM frame of a recently accelerated star, disk particles experience a torque that tends to align their orbit planes so that after relaxation, the disk angular momentum vector will tend to be at right angles to the stellar proper motion vector.

Figure 8 shows three panels from the 3D simulations described by Bate et al. (2002a, 2002b). A dynamically ejected star moves from a cluster located in the lower right of the figure to the upper left. The original disk is reoriented by the acceleration so that its spin axis is nearly orthogonal to the proper motion vector.

The expected relationship between disk orientation and proper motion can identify stars ejected by a dynamical decay of a multiple system. Disk major axes pointing to a common location may indicate dynamical decay. Lower-mass stars can be ejected with speeds of only a few kilometers per second (Reipurth et al. 2010; Reipurth \& Mikkola 2012, 2015). Disk orientations and proper motions can be measured with millimeter and submillimeter interferometers such as ALMA, NOEMA, or SMA, or using visual and infrared scattered light measurements (Jiang et al. 2005) combined with astrometry.

\section{Summary and Conclusions}

The main results from this study are:

(1) Inspection of multiple archival infrared images confirm the high proper motion of the moderate-mass young star,
Src $x$, away from the point of origin of the OMC1 explosion. These data show that in the infrared, Src n does not exhibit any measurable motion, confirming the proper motion detected at radio wavelengths likely traces a small-scale one-sided ejection of plasma. Although saturated in the IR images, BN also can be seen to move toward the northwest as shown by much more precise radio measurements.

(2) Analysis of the proper motion of the ejected stars BN, Src I, and Src x, combined with the requirement that the CoM of the three stars move with a speed less than the escape speed from OMC1, shows that Src I is likely to have a mass around $15 M_{\odot}$, consistent with recent measurements of its disk rotation curve (Ginsburg et al. 2018).

(3) The measured radial velocities of Src I and $\mathrm{BN}$, combined with the requirement that the CoM of the three ejected stars move with a speed less than the escape speed from $\mathrm{OMC} 1$, indicates that Src $\mathrm{x}$ is likely to have a radial velocity of $V_{\mathrm{LSR}} \approx-23 \pm 10 \mathrm{~km} \mathrm{~s}^{-1}$.

(4) The sizes, masses, and orientations of the Src I and BN disks, and limits on the Src x disk, are consistent with the dynamical ejection scenario. Matter orbiting the accelerated star with a Kepler speed larger than the stellar ejection speed will tend to be dragged along. Thus, the current disks are likely to be inner parts of preexisting disks that survived the dynamic ejection $\sim 550 \mathrm{yr}$ ago.

(5) The high proper motion, compact radio sources dubbed "IRc 23" and Zapata 11 by Dzib et al. (2017), and called E2 and SW1, here do not contain compact the X-ray, IR, or submillimeter sources at the sensitivity limit of existing data. Thus, they likely contribute negligible mass and momentum to the motion of the CoM analysis.

(6) These slightly extended, free-free emitting radio knots are part of a chain of fainter radio sources that traces a collimated outflow from BN younger than $500 \mathrm{yr}$ old, with a mass-loss rate of $\dot{M}>10^{-7} M_{\odot} \mathrm{yr}^{-1}$. As this ejecta moves through the dense molecular gas located behind the Orion Nebula, it is likely to be collisionally ionized by shocks with speeds of $30-50 \mathrm{~km} \mathrm{~s}^{-1}$, consistent with proper motion measurements. 
This work was supported in part by National Science Foundation (NSF) grants AST-1009847, AST-1313188, and AST-1910393. This paper makes use of the following ALMA data: ADS/JAO.ALMA \#2013.1.00546.S ALMA is a partnership of ESO (representing its member states), NSF (USA), and NINS (Japan), together with NRC (Canada), MOST and ASIAA (Taiwan), and KASI (Republic of Korea), in cooperation with the Republic of Chile. The Joint ALMA Observatory is operated by ESO, auI/NRAO and NAOJ. The National Radio Astronomy Observatory is a facility of the National Science Foundation operated under cooperative agreement by Associated Universities, Inc. We thank Professor Norio Kaifu for providing a FITS copy of the Subaru $\mathrm{H}_{2}$ image from Kaifu et al. (2000). Some of the data used in the analysis is based on observations obtained with the Apache Point Observatory $3.5 \mathrm{~m}$ telescope, which is owned and operated by the Astrophysical Research Consortium. We thank the referee, Dr. Jonathan Tan, for helpful comments which improved the manuscript.

Facilities: Apache Point Observatory, Subaru, Gemini South, ALMA, JVLA.

Software: CASA (McMullin et al. 2007), IRAF (Tody 1986, 1993), PyRAF (Science Software Branch at STScI 2012), SAO Image ds9 (Joye \& Mandel 2003).

\section{ORCID iDs}

John Bally (ib https://orcid.org/0000-0001-8135-6612 Adam Ginsburg (iD https://orcid.org/0000-0001-6431-9633 Jan Forbrich (i) https://orcid.org/0000-0001-8694-4966

\section{References}

Allen, D. A., \& Burton, M. G. 1993, Natur, 363, 54

Bally, J. 2016, ARA\&A, 54, 491

Bally, J., Cunningham, N. J., Moeckel, N., et al. 2011, ApJ, 727, 113

Bally, J., Ginsburg, A., Arce, H., et al. 2017, ApJ, 837, 60

Bally, J., Ginsburg, A., Silvia, D., \& Youngblood, A. 2015, A\&A, 579, A130

Bate, M. R., Bonnell, I. A., \& Bromm, V. 2002a, MNRAS, 332, L65

Bate, M. R., Bonnell, I. A., \& Bromm, V. 2002b, MNRAS, 336, 705

Beuther, H., Linz, H., Bik, A., et al. 2010, A\&A, 512, A29

Chuss, D. T., Andersson, B.-G., Bally, J., et al. 2019, ApJ, 872, 187

Condon, J. J., \& Ransom, S. M. 2016, Essential Radio Astronomy (Princeton, NJ: Princeton Univ. Press)

Cunningham, N. J., Moeckel, N., \& Bally, J. 2009, ApJ, 692, 943

De Buizer, J. M., Morris, M. R., Becklin, E. E., et al. 2012, ApJL, 749, L23

Dicker, S. R., Mason, B. S., Korngut, P. M., et al. 2009, ApJ, 705, 226

Draine., B. T. (ed.) 2011, Physics of the Interstellar and Intergalactic Medium (Princeton, NJ: Princeton Univ. Press)
Dzib, S. A., Loinard, L., Rodríguez, L. F., et al. 2017, ApJ, 834, 139 Farias, J. P., \& Tan, J. C. 2018, A\&A, 612, L7

Forbrich, J., Rivilla, V. M., Menten, K. M., et al. 2016, ApJ, 822, 93

Ginsburg, A., Bally, J., Goddi, C., Plambeck, R., \& Wright, M. 2018, ApJ, 860,119

Ginsburg, A., McGuire, B., Plambeck, R., et al. 2019, ApJ, 872, 54

Goddi, C., Humphreys, E. M. L., Greenhill, L. J., Chandler, C. J., \& Matthews, L. D. 2011, ApJ, 728, 15

Gómez, L., Rodríguez, L. F., Loinard, L., et al. 2008, ApJ, 685, 333

Grosso, N., Feigelson, E. D., Getman, K. V., et al. 2005, ApJS, 160, 530

Hirota, T., Kim, M. K., Kurono, Y., \& Honma, M. 2014, ApJL, 782, L28

Hirota, T., Machida, M. N., Matsushita, Y., et al. 2017, NatAs, 1, 0146

Indriolo, N., Tan, J. C., Boogert, A. C. A., et al. 2018, ApJL, 865, L18

Jiang, Z., Tamura, M., Fukagawa, M., et al. 2005, Natur, 437, 112

Joye, W. A., \& Mandel, E. 2003, in ASP Conf. Ser. 295, Astronomical Data Analysis Software and Systems XII, ed. H. E. Payne, I. Jedrzejewski, \& R. N. Hook (San Francisco, CA: ASP)

Kaifu, N., Usuda, T., Hayashi, S. S., et al. 2000, PASJ, 52, 1

Kim, D., Lu, J. R., Konopacky, Q., et al. 2019, AJ, 157, 109

Kim, M. K., Hirota, T., Honma, M., et al. 2008, PASJ, 60, 991

Kim, M. K., Hirota, T., Machida, M. N., et al. 2019, ApJ, 872, 64

Kounkel, M., Hartmann, L., Loinard, L., et al. 2017, ApJ, 834, 142

Lane, J., Kirk, H., Johnstone, D., et al. 2016, ApJ, 833, 44

Lonsdale, C. J., Becklin, E. E., Lee, T. J., \& Stewart, J. M. 1982, AJ, 87, 1819

Luhman, K. L., Robberto, M., Tan, J. C., et al. 2017, ApJL, 838, L3

Matthews, L. D., Greenhill, L. J., Goddi, C., et al. 2010, ApJ, 708, 80

McMullin, J. P., Waters, B., Schiebel, D., et al. 2007, in ASP Conf. Ser. 376, Astronomical Data Analysis Software and Systems XVI, ed. R. A. Shaw et al. (San Francisco, CA: ASP)

Menten, K. M., Reid, M. J., Forbrich, J., \& Brunthaler, A. 2007, A\&A, 474,515

Moeckel, N., \& Bally, J. 2006, ApJ, 653, 437

Moeckel, N., \& Bally, J. 2007, ApJ, 656, 275

O’Dell, C. R. 2001, ARA\&A, 39, 99

Plambeck, R. L., Bolatto, A. D., Carpenter, J. M., et al. 2013, ApJ, 765, 40

Plambeck, R. L., \& Wright, M. C. H. 2016, ApJ, 833, 219

Reipurth, B., \& Mikkola, S. 2012, Natur, 492, 221

Reipurth, B., \& Mikkola, S. 2015, AJ, 149, 145

Reipurth, B., Mikkola, S., Connelley, M., et al. 2010, ApJL, 725, L56

Rodríguez, L. F., Dzib, S. A., Loinard, L., et al. 2017, ApJ, 834, 140

Scoville, N., Kleinmann, S. G., Hall, D. N. B., \& Ridgway, S. T. 1983, ApJ, 275,201

Scoville, N. Z., Hall, D. N. B., Kleinmann, S. G., et al. 1979, ApJL, 232, L121

Shuping, R. Y., Morris, M., \& Bally, J. 2004, AJ, 128, 363

Smith, N., Bally, J., Shuping, R. Y., Morris, M., \& Kassis, M. 2005, AJ, 130, 1763

Tody, D. 1986, Proc. SPIE, 627, 733

Tody, D. 1993, in ASP Conf. Ser. 52, Astronomical Data Analysis Software and Systems II, ed. R. J. Hanisch, R. J. V. Brissenden, \& J. Barnes (San Francisco, CA: ASP)

Zapata, L. A., Rodríguez, L. F., Kurtz, S. E., et al. 2004, AJ, 127, 2252

Zapata, L. A., Schmid-Burgk, J., Ho, P. T. P., Rodríguez, L. F., \& Menten, K. M. 2009, ApJL, 704, L45

Zapata, L. A., Schmid-Burgk, J., Rodríguez, L. F., Palau, A., \& Loinard, L. 2017, ApJ, 836, 133 